\title{
Répteis do Estado de São Paulo: conhecimento atual e perspectivas
}

\author{
Hussam Zaher ${ }^{1,6}$, Fausto Erritto Barbo ${ }^{1,2}$, Paola Sanchez Martínez, \\ Cristiano Nogueira ${ }^{3}$, Miguel Trefaut Rodrigues ${ }^{4} \&$ Ricardo Jannini Sawaya $^{5}$ \\ ${ }^{1}$ Museu de Zoologia, Universidade de São Paulo - USP, \\ Av. Nazaré, n. 481, Ipiranga, CEP 04263-000, São Paulo, SP, Brasil \\ ${ }^{2}$ Programa de Pós-graduação em Biologia Animal, Instituto de Biociências, Letras e Ciências Exatas, \\ Universidade Estadual Paulista - UNESP, Rua Cristóvão Colombo, CP 2265, CEP 15054-000, \\ São José do Rio Preto, SP, Brasil \\ ${ }^{3}$ Departamento de Zoologia, Universidade de Brasília - UnB, CEP 70910-900, Brasília, DF, Brasil \\ ${ }^{4}$ Departamento de Zoologia, Instituto de Biociências, Universidade de São Paulo - USP, \\ Rua do Matão Travessa 14, Cidade Universitária, CEP 05508-900, São Paulo, SP, Brasil \\ ${ }^{5}$ Departamento de Ciências Biológicas, Universidade Federal de São Paulo - UNIFESP, \\ Rua Prof. Artur Riedel, n. 275, Eldorado, CEP 09972-270, Diadema, SP, Brasil \\ ${ }^{6}$ Autor para correspondência: Hussam Zaher,e-mail: hzaher@usp.br
}

ZAHER, H., BARBO, F.E., MARTÍNEZ, P.S., NOGUEIRA, C., RODRIGUES, M.T. \& SAWAYA R.J. Reptiles from São Paulo State: current knowledge and perspectives. Biota Neotrop. 11(1a): http://www.biotaneotropica. org.br/v11n1a/en/abstract?inventory+bn0051101a2011.

\begin{abstract}
The present study provides a list of species of reptiles known to occur in the State of São Paulo, based on specimens with confirmed localities, housed in the three largest scientific collections of the state, and published information. We registered the presence of 212 species of reptiles within the boundaries of the state that belong to 23 families and include 12 chelonians, three crocodilians, and 197 squamates (142 snakes, 44 "lizards," and 11 amphisbenians). Eleven species are endemic to the State of São Paulo (Mesoclemmys cf. vanderhaegei, Amphisbaena sanctaeritae, Mabuya caissara, Mabuya macrorhyncha, Liotyphlops caissara, Liotyphlops schubarti, Corallus cropanii, Atractus serranus, Phalotris lativittatus, Bothropoides alcatraz, Bothropoides insularis). Within Squamata, four lizards and one snake correspond to valid species that are still in need of formal description. Thirtytwo species are included in the List of Threatened Species from the State of São Paulo, while only nine belong to the Brazilian List of Threatened Species. Reptile species richness for the state represents already approximately $30 \%$ of species richness known to occur in all the Brazilian territory, which includes 721 species. However, we conclude that more detailed taxonomic and distributional studies as well as more surveys targeting poorly known localities will have the effect of augmenting the number of species for the state.

Keywords: reptiles, biodiversity, State of São Paulo, BIOTA/FAPESP program.
\end{abstract}

Number of species: In the world: 8,734 (Uetz 2010), in Brazil: 721 (Bérnils 2010), estimated in São Paulo State: 250.

ZAHER, H., BARBO, F.E., MARTÍNEZ, P.S., NOGUEIRA, C., RODRIGUES, M.T. \& SAWAYA R.J. Répteis do Estado de São Paulo: conhecimento atual e perspectivas. Biota Neotrop. 11(1a): http://www.biotaneotropica. org.br/v11n1a/pt/abstract?inventory+bn0051101a2011.

Resumo: Elaboramos uma lista das espécies de répteis do Estado de São Paulo com base nos registros confirmados de exemplares depositados nas três maiores coleções científicas do estado, complementando esta informação com a bibliografia disponível. Registramos a presença de 212 espécies de répteis no Estado de São Paulo, distribuídas em 23 famílias, incluindo 12 quelônios, três crocodilianos e 197 Squamata (142 serpentes, 44 "lagartos" e 11 anfisbenas). Destas, onze são endêmicas do Estado de São Paulo (Mesoclemmys cf. vanderhaegei, Amphisbaena sanctaeritae, Mabuya caissara, Mabuya macrorhyncha, Liotyphlops caissara, Liotyphlops schubarti, Corallus cropanii, Atractus serranus, Phalotris lativittatus, Bothropoides alcatraz, Bothropoides insularis). Entre os Squamata, quatro lagartos e uma serpente correspondem a espécies ainda não descritas. Destas 212 espécies, 32 estão incluídas na Lista das Espécies Ameaçadas do Estado de São Paulo, enquanto que apenas nove constam na Lista das Espécies Ameaçadas do Brasil. A riqueza de répteis atualmente registrada no Estado de São Paulo representa cerca de $30 \%$ da riqueza conhecida para o grupo em todo o território brasileiro, que abrange 721 espécies. Entretanto, concluímos que estudos mais detalhados sobre a taxonomia e a distribuição de espécies bem como os esforços de coleta em áreas com lacunas de amostragem devem ainda aumentar o número de espécies de répteis no estado.

Palavras-chave: répteis, biodiversidade, Estado de São Paulo, programa BIOTA/FAPESP. 


\section{Introdução}

Os "répteis" atuais compreendem os clados Testudines (tartarugas, cágados e jabutis), Crocodilia (crocodilos e jacarés) e Squamata (lagartos, anfisbenas e serpentes), todos pertencentes à irradiação monofilética dos Sauropsida (ou Reptilia) que também inclui as aves. Squamata é o grupo mais diversificado entre os répteis atuais.

São conhecidas no mundo 8.734 espécies de répteis (Uetz 2010). No Brasil, foram registradas até o momento 721 espécies (Bérnils 2010), representando pouco mais de $8 \%$ do total conhecido para o planeta. Apesar de elevados, estes números não refletem a real diversidade de répteis do Brasil, que ainda conta com um número significativo de espécies por descrever. Nos últimos anos, o crescente esforço amostral desempenhado por diversos grupos de pesquisa em todo o território nacional evidenciou as lacunas de informação ainda existentes, além de melhorar substancialmente o conhecimento que hoje temos sobre os répteis do país. Entre estas iniciativas, destaca-se a do Programa Biota FAPESP que nos últimos dez anos estimulou uma ação concentrada que resultou na elaboração de um quadro bem mais detalhado da biodiversidade do Estado de São Paulo.

O Estado de São Paulo possui 3.457.301 ha remanescentes de cobertura vegetal natural, que correspondem a $13,9 \%$ da sua superfície, distribuídos de acordo com as seguintes porcentagens para cada fitofisionomia, todas inseridas nos biomas de Mata Atlântica e Cerrado (Kronka et al. 2005): 5,8\% de floresta, 5,9\% de capoeira, $0,6 \%$ de cerrado, $0,3 \%$ de cerradão, menos de $0,1 \%$ de campo cerrado, menos de $0,1 \%$ de campo, $0,6 \%$ de vegetação de várzea, $0,1 \%$ de mangue e $0,6 \%$ de restinga. Os mesmos autores constataram que as áreas de Cerrado sofreram drástica redução no estado entre 1962 e 2005 , com a supressão de $88,5 \%$ de sua cobertura original, em função da expansão agrícola e do reflorestamento com espécies exóticas para a indústria de madeira e celulose. A cobertura de Mata Atlântica do estado foi também significativamente reduzida pelos mesmos atores, a partir de um longo processo de exploração e degradação em escala nacional que resultou na redução para apenas 11 a $16 \%$ de sua cobertura original em todo o país (Ribeiro et al. 2009). A acelerada taxa de conversão das áreas naturais em pastagens e monoculturas bem como o alto grau de fragmentação dos remanescentes naturais do estado representam os dois principais problemas enfrentados no esforço pela sua conservação.

Tanto a diversidade quanto a distribuição dos répteis do Estado de São Paulo ainda são mal compreendidas e não contam com uma base de dados que seja representativa para o grupo na região. Uma revisão recente (Rossa-Feres et al. 2008) aponta que o Estado de São Paulo abriga cerca de 200 espécies de répteis, o que corresponderia a cerca de $30 \%$ das espécies de répteis conhecidas no Brasil.

A Lista Nacional das Espécies da Fauna Brasileira Ameaçadas de Extinção (Instituto... 2008) inclui mais de 120 espécies que ocorrem no Estado de São Paulo. Entre elas, há nove répteis (Caretta caretta, Chelonia mydas, Eretmochelys imbricata, Lepidochelys olivacea, Dermochelys coriacea, Corallus cropanii, Dipsas albifrons, Bothropoides alcatraz, Bothropoides insularis), embora o número seja provavelmente maior (cf. Marques et al. 2006, Sawaya et al. 2008). Répteis podem ser considerados indicadores de qualidade ambiental (Pough et al. 2004, Rossa-Feres et al. 2008) e em várias regiões do planeta tem sido registrado o declínio de suas populações, geralmente em decorrência da perda de hábitats naturais (Gibbons et al. 2000). Por apresentarem distribuição geográfica relativamente restrita quando comparada à de outros grupos de vertebrados terrestres (Gaston 1996), os estudos biogeográficos com répteis podem auxiliar na identificação de áreas de endemismo (ver exemplos em Vanzolini \& Williams 1981, Rodrigues 1986), fundamentais para compreender os processos de especiação (Eken et al. 2004, Raxworthy et al. 2003).
Uma revisão recente da lista estadual de répteis ameaçados de extinção do Estado de São Paulo considera 33 espécies como ameaçadas, o que corresponde a cerca de $15 \%$ da fauna do estado (Marques et al. 2009a). Entretanto, o status de conservação da boa parte das populações de répteis que ocorrem no estado ainda é pouco conhecido. Uma avaliação satisfatória sobre esta questão somente será possível a partir do momento em que os conhecimentos básicos sobre a identidade e distribuição dessas espécies estiverem adequadamente levantados. Mapeamentos mais detalhados de ambientes remanescentes, estudos de modelagem de nicho ecológico visando descrever regiões de potencial ocorrência e inventários em áreas protegidas são ações necessárias para uma melhor compreensão da diversidade pretérita e atual no Estado de São Paulo.

Apresentamos aqui uma lista atualizada das espécies de répteis que ocorrem no Estado de São Paulo. Essas informações, além de contribuírem para o aumento do conhecimento sobre a diversidade e distribuição dos répteis do sudeste do Brasil, são essenciais para a elaboração e avaliação de políticas de conservação e manejo da fauna de répteis do estado, assim como para definição de áreas prioritárias para sua conservação.

\section{Métodos}

A lista de espécies foi baseada na literatura disponível e em registros confirmados de exemplares depositados nas seguintes coleções científicas do estado: Museu de Zoologia da Universidade de São Paulo (MZUSP), Instituto Butantan (IBSP) e Museu de Zoologia da Universidade Estadual de Campinas (ZUEC). Estas correspondem às três coleções mais representativas dos répteis do Estado de São Paulo, nas quais ingressou nos últimos anos um número significativo de exemplares oriundos principalmente do esforço de coleta empreendido pelo Programa BIOTA/FAPESP. Os espécimes testemunho utilizados para a conclusão deste trabalho foram analisados e identificados por um ou mais autores, ou ainda, quando necessário, confirmados com o auxílio de especialistas. Não utilizamos registros provenientes de bases de dados eletrônicas disponíveis na internet, que muitas vezes replicam e disponibilizam informações brutas e não revisadas, resultando em interpretações equivocadas sobre padrões de diversidade e distribuição.

A distribuição das espécies foi determinada a partir do georeferenciamento dos registros confirmados dos exemplares depositados nas três coleções examinadas, descartando assim os registros da literatura para os quais não foi possível confirmar a presença e identificação de espécime testemunho. A revisão detalhada e o exame direto do material tombado, com extenso trabalho de correção de registros, georeferenciamento de localidades e eliminação de dados duvidosos, é um passo crucial em todo e qualquer estudo de síntese em biodiversidade. Esta abordagem conduziu à elaboração de uma lista confiável de registros que poderão ser conferidos individualmente e de forma independente, fornecendo assim uma base sólida para a elaboração de mapas de distribuição cada vez mais completos, fidedignos e detalhados das espécies que ocorrem no estado.

Os registros da literatura foram empregados apenas nos casos confiáveis em que a espécie foi assinalada para o estado, mas não conta com exemplar tombado nas coleções analisadas. Das 212 espécies que compõem a lista, apenas sete entram nesta categoria e não contam com espécimes testemunho nas coleções analisadas: Acanthochelys radiolata, Eretmochelys imbricata, Lepidochelys olivacea, Dermochelys coriacea, Trachemys dorbigni e Caiman yacare. Entretanto, essas espécies contam com registros visuais confiáveis e, portanto, foram incluídas na lista. 
Esta lista não inclui as subespécies reconhecidas na literatura por se tratarem de categoria taxonômica conceitual e operacionalmente questionável (Frost \& Hillis 1990). As espécies exóticas registradas no estado (cf. Eterovic \& Duarte 2002, Marques et al. 2009a) também não foram incluídas na presente lista.

Para a realização de uma análise de lacunas de amostragem, analisamos os registros de ocorrência das espécies de répteis no programa DIVA-GIS (Hijmans et al. 2001), através da interpolação circular neighborhood (Cressie, 1991). O produto dessa análise é um mapa em formato rasterizado, que aponta as regiões do estado com maior representatividade de registros em coleções, assim como aquelas que apresentam lacunas de amostragens.

As espécies também foram caracterizadas em relação à sua ocorrência nas diferentes formações vegetais do estado. Essas informações foram obtidas utilizando um shapefile das formações de vegetação do Estado de São Paulo (Metzger et al. 2008) e a ferramenta extract values by points, no programa DIVA-GIS (Hijmans et al. 2001).

Para ilustrar a taxa de descrição de espécies de répteis no Estado de São Paulo desde Linnaeus até 2010, ajustamos uma curva polinomial aos pontos cumulativos correspondentes a cada descrição de uma nova espécie (Figura 1), empregando como referência o ano de publicação desta. As espécies que ainda não foram descritas formalmente mas que estão registradas na presente lista foram acrescentadas à curva. Além de ilustrar a taxa de descrição de espécies na região, esta curva também indica se todas as espécies que devem ocorrer no estado já foram registradas ou descritas. Caso a curva ainda apresente uma inclinação diferente de zero nos últimos anos, pode-se considerar que novas espécies deverão ser registradas ou descritas para o estado futuramente. Consideramos apenas os Squamata para a confecção da curva, por estes constituírem um grupo monofilético e representarem a grande maioria das espécies do grupo $(\mathrm{N}=197)$.

Seguimos as propostas de classificação sugeridas por Ernst \& Barbour (1989) e Bour \& Zaher (2005) para Testudines, Brazaitis (1973) e Busack \& Pandya (2001) para Crocodilia, e Pough et al. (2004), Pellegrino et al. (2001), Frost et al. (2001), Conrad (2008), Gamble et al. (2008), Fenwick et al. (2009), Mott \& Vieites (2009) e Zaher et al. (2009) para Squamata. Adotamos também a proposta taxonômica para serpentes elaborada por Zaher et al. (2009) no que



Figura 1. Curva de acumulação de espécies gerada a partir do ano de descrição original ou ano de reconhecimento de espécies novas ainda não descritas de répteis Squamata (pontos escuros) registrados no estado de São Paulo, entre os anos de 1758 e 2010.

Figure 1. Cumulative curve of species based on the year of original description of the species or the year in which a new species was informally recognized (dark dots) in the state of São Paulo, from 1758 to 2010. diz respeito aos gêneros Liophis Wagler 1830, Erythrolamprus Boie 1826 e Lygophis Fitzinger 1843. Entretanto, com exceção das espécies incluídas em Lygophis e Caaeteboia por Zaher et al. (2009), as demais espécies são alocadas aqui no gênero Erythrolamprus Boie, 1826 (veja Forlani et al. 2010). Foram incluídas na lista algumas formas que correspondem a espécies ainda não descritas ou em processo de descrição. Quanto à subdivisão do gênero Bothrops sugerida por Fenwick et al. (2009), apesar de compartilharmos das ressalvas feitas por Carrasco et al. (2009, 2010), não resta outra opção que não seja reconhecer os gêneros Bothropoides e Rhinocerophis no presente trabalho, até que um análise mais abrangente das relações filogenéticas no grupo seja concluída.

No caso do gênero Placosoma, reconhecemos como espécies plenas as subespécies de Placosoma cordylinum empregadas por Uzzell (1959) pois sua ocorrência simpátrica e os caracteres que as diagnosticam apóiam este novo status. Anolis nitens (Wagler, 1840) foi descrito sumariamente e sem localidade precisa. Em razão da descrição insuficiente apresentada por Wagler, Vanzolini \& Williams (1970) passaram a utilizar Anolis chrysolepis para este complexo de espécies. Ávila-Pires (1995) voltou a A. nitens e, desde então, ambos os nomes (A. nitens e A. chrysolepis) passaram a ser empregados sem muito critério e seguindo a preferência dos autores. A tendência atual tem sido manter $A$. chrysolepis com base em proposta avançada por Myers (2009), e submetida à CINZ, para supressão de $A$. nitens Seguimos aqui Myers (2009) ao empregar apenas o nome Anolis chrysolepis.

A situação de Mabuya bistriata e M. nigropunctata é mais complicada. Williams \& Vanzolini (1980) utilizaram sem justificativa Mabuya bistriata (Spix, 1825) para os lagartos até então identificados como Mabuya mabouya, alegando que o grupo estava sendo revisto por Rebouças-Spieker. Mais tarde, estudando a variação de lagartos do gênero na Amazônia, Rebouças-Spieker reconheceu duas espécies e à maior delas atribuiu o nome de Mabuya bistriata por semelhança com a descrição, a prancha e o itinerário de Spix (Rebouças-Spieker, 1981a). Embora se acreditasse que os tipos estivessem perdidos, a autora não designou um neótipo nem tampouco propôs a figura de Spix como lectótipo. Vários outros trabalhos passaram a empregar M. bistriata seguindo esse conceito (Rebouças-Spieker, 1981a,b, Vanzolini 1981a,b, Vanzolini \& Williams, 1981). A outra espécie foi descrita como Mabuya ficta por Rebouças-Spieker em um segundo trabalho do mesmo ano (Rebouças-Spieker, 1981b). Hoogmoed \& Gruber (1983), adotaram este novo conceito e, pretendendo estabilizar a nomenclatura, designaram um lectótipo para Mabuya bistriata entre o material da série tipo de Spix, então redescoberto. Contudo, por uma falha na identificação, este tratava-se de um espécime de $M$. ficta e não do que vinha sendo reconhecido por M. bistriata. Ávila-Pires (1995) percebeu o engano, sinonimizou $M$. ficta a $M$. bistriata e sugeriu que outro dos nomes esquecidos de Spix, agora $M$. nigropunctata, também sem localidade definida e sem tipos, passasse a ser o nome utilizado para a espécie que vinha até então sendo tratada por $M$. bistriata. Para tal designou um neótipo para Mabuya nigropunctata, que se assemelhava à descrição de Spix. Este é o cenário taxonômico confuso que explica a razão pela qual as mabuias deste complexo vêm sendo tratadas ora por $M$. nigropunctata ora por M. bistriata. Embora saibamos que este é um complexo bastante diversificado de espécies (Miralles \& Carranza 2010, Whiting et al. 2006), é seguro dizer que M. bistriata, conforme concebida atualmente, não ocorre fora da Amazônia. Para as espécies do complexo que ocorrem na Amazônia, no Cerrado, na Mata Atlântica e em habitats especiais pertencentes ao domínio das Caatingas, adotamos aqui $M$. nigropunctata como nome de conveniência. Não temos dúvida, contudo, de que os espécimes de São Paulo serão futuramente reconhecidos com outro nome. 


\section{Resultados e Discussão}

\section{Lista das espécies de répteis do Estado de São Paulo}

Foram registradas 212 espécies de répteis no Estado de São Paulo, incluindo 12 quelônios, três crocodilianos e 197 Squamata (142 serpentes, 44 "lagartos" e 11 anfisbenas; Tabela 1). Destas, onze são endêmicas do estado (Tabela 1). As três espécies de crocodilianos pertencem à mesma família Alligatoridae, enquanto que as 12 espécies de quelônios estão distribuídas em quatro famílias distintas (Chelidae, Cheloniidae, Dermochelyidae, Emydidae). Os Squamata contam com 18 famílias ao todo (Amphisbaenidae, Anguidae, Leiosauridae, Phyllodactylidae, Polychrotidae, Tropiduridae, Scincidae, Gymnophthalmidae, Teiidae, Anomalepididae, Leptotyphlopidae, Typhlopidae, Tropidophiidae, Boidae, Colubridae, Dipsadidae, Elapidae, Viperidae). Entre os Squamata, quatro lagartos e uma serpente correspondem a espécies ainda não descritas. Das 212 espécies registradas no presente trabalho, 32 estão incluídas na Lista das Espécies Ameaçadas do Estado de São Paulo (Marques et al. 2009a), enquanto que apenas nove pertencem também à Lista das Espécies Ameaçadas do Brasil (Ministério... 2003).

\section{A riqueza de répteis do Estado do São Paulo e principais lacunas de conhecimento}

Embora o ritmo de descrição de novas espécies de répteis tenha crescido consideravelmente nas duas últimas décadas (Figura 1) no Estado de São Paulo, a inclinação da curva de acumulação indica que um número significativo de espécies ainda deve ser descrito ou ter sua distribuição registrada no estado. Embora esses dados não permitam estimativas confiáveis ou objetivas da riqueza real de espécies, sugerimos que um total de pelo menos 250 espécies de répteis ocorram no estado. Isto denota a necessidade de intensificar os estudos de taxonomia, sistemática e distribuição geográfica dos répteis de São Paulo. Há outras razões para se pensar desta forma. O amplo desmatamento sofrido pelo estado no século passado eliminou definitivamente importantes áreas de Cerrado encravados em paisagens florestais que evoluíram isoladamente da área central do domínio. Essas paisagens, já espacialmente pouco expressivas quando intactas, praticamente desapareceram com o avanço da agricultura, passando a fazer parte de um mosaico de ambientes antropizados que, descaracterizados, atraem pouco o interesse dos zoólogos. No entanto, por terem evoluído em isolamento e por sua fauna estar adaptada às áreas abertas, é possível que ainda abriguem endemismos restritos de grande importância histórica e biogeográfica. Os capões de mata isolados nos cerrados do estado, bem como os topos de montanhas elevadas cobertos por vegetação florestal ou aberta, podem representar exemplos semelhantes. O potencial de preservação da biodiversidade regional e dos seus endemismos, presente em tais ambientes, aponta para a necessidade urgente de intensificar as pesquisas de campo em toda a área de ocorrência do Cerrado e da Mata Atlântica, possibilitando assim a construção de uma visão mais ampla da diversidade no estado e no país em médio prazo.

O levantamento de dados de coleções, literatura e expedições de coleta, forneceu um enorme volume de informação que revelou a atual situação do conhecimento sobre a fauna de répteis do estado de São Paulo (Figura 2). O mapa da Figura 2 ilustra as áreas com alta representatividade de répteis nas coleções, e também aponta para as áreas carentes de estudos e de levantamentos detalhados de espécies no estado. Uma ampla parcela do território do estado está bem amostrada nas coleções. A área mais significativamente amostrada ocupa quase toda a metade do estado, englobando a Serra do Mar (todo o Vale do Ribeira, regiões de Itanhaém, Itapecerica da Serra, São Paulo, Santos, Mogi das Cruzes, São José dos Campos,
Caraguatatuba, Paraibuna, São Luis do Paraitinga, Ubatuba e Guaratinguetá) e as regiões sudeste, nordeste e central do estado (grande São Paulo, regiões de Piedade, Sorocaba, Itapetininga, Botucatu, Piracicaba, Bauru, Jaú, Araraquara, Rio Claro, São Carlos, Mogi-Mirim, Pirassununga, Ribeirão Preto e Campinas). Essa área com elevada concentração de registros de espécies abrange um raio de aproximadamente $300 \mathrm{~km}$ ao redor da cidade de São Paulo. Esta elevada representatividade se deve especialmente ao grande número de expedições realizadas ao longo do século XX e na primeira década do século XXI, mas também ao encaminhamento constante de serpentes capturadas pelos moradores locais, estimulados pelas permutas de soro mantidas pelo Instituto Butantan.

Nesta área de maior conhecimento, contamos com inventários bastante completos de répteis provenientes da cidade de São Paulo (cf. Marques et al. 2009b, Barbo 2008, Barbo \& Sawaya 2008), dos municípios da Serra do Mar (cf. Marques 1998, Cicchi et al. 2007, Condez et al. 2009, Hartmann et al. 2009, Forlani et al. 2010) e da região mais central do estado (Vanzolini 1948, Sawaya et al. 2008, Araújo et al. 2010). A área mais a noroeste, localizada na divisa com o Estado de Mato Grosso do Sul, conta também com uma grande representatividade de exemplares provenientes do enchimento do reservatório da usina hidrelétrica de Ilha Solteira.

A área que apresentou baixa representatividade em coleções (lacunas de amostragem) ocupa uma parcela menor do território do estado, se distribuindo pelo norte e o noroeste do estado, onde estão localizados os municípios de São José do Rio Preto, Lins, Birigui, Tupã e Marília, e estendendo para o oeste na região de Presidente Prudente, para o nordeste na região de São João da Boa Vista, e para o sudoeste na região de Ourinhos, Itapeva e Capão Bonito (localizados na divisa com o estado do Paraná). Estas áreas pouco representadas em coleções podem ser indicadas como prioritárias na elaboração de futuros projetos de pesquisa que visam o levantamento de espécies de répteis.

\section{A riqueza de répteis do Estado do São Paulo e de outras regiões}

A riqueza de répteis detectada no Estado de São Paulo (212 espécies) representa cerca de $30 \%$, ou um pouco menos de um terço, da riqueza de répteis conhecida para todo o território brasileiro (721 espécies; Bérnils, 2010). Considerando que a área do Estado de São Paulo $\left(248.209 \mathrm{~km}^{2}\right)$ não excede $3 \%$ de todo o território brasileiro $\left(8.514 .876 \mathrm{~km}^{2}\right)$, fica claro que o estado apresenta riqueza desproporcionalmente elevada em relação à área que ocupa. A alta riqueza documentada no estado certamente está relacionada à diversidade de formas de paisagem presentes na região (Tabela 1). Embora ainda sejam necessários estudos mais amplos, esta comparação demonstra que São Paulo corresponde a uma das porções do território nacional com a diversidade de répteis mais bem inventariada do país. Este resultado se deve aos esforços constantes e duradouros das instituições de pesquisa e de fomento e à existência e manutenção das coleções zoológicas do estado.

A riqueza no estado de São Paulo é também bastante similar à detectada em compilação recente para o estado de Minas Gerais, que abriga 221 espécies (nove quelônios, três crocodilianos, 57 lagartos, 13 anfisbenas e 139 serpentes; Bérnils et al. 2009). Embora haja uma similaridade marcante na herpetofauna destas duas regiões contíguas, que compartilham grande parte de suas unidades biogeográficas (e. g. planaltos da alta bacia platina, Serra da Mantiqueira) e são recobertas pelos mesmos domínios de vegetação (Cerrado e Mata Atlântica), Minas Gerais possui área cerca de duas vezes maior do que a do Estado de São Paulo (526.528 km²), além de conter formações vegetais e paisagísticas ausentes em São Paulo (e. g. Caatingas, no noroeste de Minas e campos rupestres da Serra do Espinhaço). Assim, é bem provável que a riqueza e diversidade dos répteis de Minas Gerais ainda estejam bastante subestimadas. 
Tabela 1. Répteis do Estado de São Paulo e sua distribuição nas diferentes fitofisionomias. Fitofisionomias - FES: Floresta Estacional Semidecidual; FOD: Floresta Ombrófila Densa; FOM: Floresta Ombrófila Mista; CER: Cerrado; FOM/FES: área de contato entre Floresta Ombrófila Mista e Floresta Estacional Semidecidual; FOD/FOM: área de contato entre Floresta Ombrófila Densa e Floresta Ombrófila Mista; CER/FES: área de contato entre Cerrado e Floresta Estacional Semidecidual; CER/FOD: área de contato entre Cerrado e Floresta Ombrófila Densa; CER/FOM: área de contato entre Cerrado e Floresta Ombrófila Mista. Listas e categorias de ameaça às espécies - SP: espécies presentes na lista de espécies ameaçadas do Estado de São Paulo (Marques et al. 2009a); BR: espécies presentes na Lista de Espécies Ameaçadas do Brasil (Ministério do Meio Ambiente - MMA, 2003); CR: criticamente em perigo; EN: em perigo; VU: vulnerável. As espécies anotadas com (*) são endêmicas do estado.

Table 1. Reptiles and their distribution within the distinct phyto-physionomies of the state of São Paulo. Phyto-physionomies - FES: Semideciduous stationary forest; FOD: Dense ombrophilous forest; FOM: Mixed ombrophilous forest; CER: Cerrado; FOM/FES: Area of contact between Mixed ombrophilous forest and Semideciduous stationary forest; FOD/FOM: Area of contact between Dense ombrophilous forest and Mixed ombrophilous forest; CER/FES: Area of contact between Cerrado and Semideciduous stationary forest; CER/FOD: Area of contact between Cerrado and Dense ombrophilous forest; CER/FOM: Area of contact between Cerrado and Mixed ombrophilous forest. List and categories of species threat: SP: species present in the list of threatened species of the state of São Paulo (Marques et al. 2009a); BR: species present in the list of Brazilian Threatened species (Ministério do Meio Ambiente - MMA, 2003); CR: critically endangered; EN: endangered; VU: vulnerable. Species with an asterisk (*) are endemic to the state.

\begin{tabular}{|c|c|c|c|c|c|c|c|c|c|c|c|}
\hline Taxon & FES & FOD & FOM & CER & $\begin{array}{c}\text { FOM/ } \\
\text { FES }\end{array}$ & $\begin{array}{l}\text { FOD/ } \\
\text { FOM }\end{array}$ & $\begin{array}{c}\text { CER/ } \\
\text { FES }\end{array}$ & $\begin{array}{l}\text { CER/ } \\
\text { FOD }\end{array}$ & $\begin{array}{l}\text { CER/ } \\
\text { FOM }\end{array}$ & SP & $\mathbf{B R}$ \\
\hline \multicolumn{12}{|l|}{ TESTUDINES } \\
\hline \multicolumn{12}{|l|}{ Chelidae } \\
\hline Acanthochelys radiolata (Mikan, 1820) & - & $\mathrm{X}$ & - & - & - & - & - & - & - & - & - \\
\hline Acantochelys spixii (Duméril \& Bibron, 1835) & - & $\mathrm{X}$ & - & - & - & - & - & - & - & - & - \\
\hline Hydromedusa maximiliani (Mikan, 1820) & $\mathrm{X}$ & $\mathrm{X}$ & - & - & - & - & - & $\mathrm{X}$ & $\mathrm{X}$ & - & - \\
\hline Hydromedusa tectifera Cope, 1869 & $\mathrm{X}$ & $\mathrm{X}$ & $\mathrm{X}$ & - & - & - & - & - & - & - & - \\
\hline Mesoclemmys cf. vanderhaegei (Bour, 1973)* & $\mathrm{X}$ & - & - & - & - & - & - & - & - & - & - \\
\hline Phrynops geoffroanus (Schweigger, 1812) & $\mathrm{X}$ & - & - & - & - & - & $\mathrm{X}$ & - & - & - & - \\
\hline \multicolumn{12}{|l|}{ Cheloniidae } \\
\hline Caretta caretta (Linnaeus, 1758) & - & - & - & - & - & - & - & - & - & VU & VU \\
\hline Chelonia mydas (Linnaeus, 1758) & - & - & - & - & - & - & - & - & - & VU & VU \\
\hline Eretmochelys imbricata (Linnaeus, 1766) & - & - & - & - & - & - & - & - & - & VU & VU \\
\hline Lepidochelys olivacea (Eschscholtz, 1829) & - & - & - & - & - & - & - & - & - & VU & VU \\
\hline \multicolumn{12}{|l|}{ Dermochelyidae } \\
\hline Dermochelys coriacea (Linnaeus, 1766) & - & - & - & - & - & - & - & - & - & $\mathrm{CR}$ & CR \\
\hline \multicolumn{12}{|l|}{ Emydidae } \\
\hline Trachemys dorbignyi (Duméril \& Bibron, 1835) & $\mathrm{X}$ & - & - & - & - & - & - & - & - & - & - \\
\hline \multicolumn{12}{|l|}{ CROCODILIA } \\
\hline \multicolumn{12}{|l|}{ Alligatoridae } \\
\hline Caiman latirostris (Daudin, 1802) & $\mathrm{X}$ & $\mathrm{X}$ & - & $\mathrm{X}$ & - & - & $\mathrm{X}$ & - & - & - & - \\
\hline Caiman yacare (Daudin, 1802) & - & - & - & - & - & - & $\mathrm{X}$ & - & - & - & - \\
\hline Paleosuchus palpebrosus (Cuvier, 1807) & - & - & - & - & - & - & $\mathrm{X}$ & - & - & - & - \\
\hline \multicolumn{12}{|l|}{ SQUAMATA - Amphisbaenia } \\
\hline \multicolumn{12}{|l|}{ Amphisbaenidae } \\
\hline Amphisbaena alba Linnaeus, 1758 & $\mathrm{X}$ & $\mathrm{X}$ & - & $\mathrm{X}$ & - & - & $\mathrm{X}$ & $\mathrm{X}$ & - & - & - \\
\hline Amphisbaena dubia L. Müller, 1924 & - & $\mathrm{X}$ & $\mathrm{X}$ & - & - & - & - & $\mathrm{X}$ & - & - & - \\
\hline Amphisbaena hogei Vanzolini, 1950 & - & $\mathrm{X}$ & - & - & - & - & - & - & - & - & - \\
\hline Amphisbaena mertensi Strauch, 1881 & $\mathrm{X}$ & $\mathrm{X}$ & $\mathrm{X}$ & $\mathrm{X}$ & - & - & $\mathrm{X}$ & $\mathrm{X}$ & - & - & - \\
\hline Amphisbaena prunicolor (Cope, 1885) & - & - & - & - & - & - & $\mathrm{X}$ & - & - & - & - \\
\hline Amphisbaena sanctaeritae Vanzolini, 1994* & $\mathrm{X}$ & - & - & - & - & - & - & - & - & VU & - \\
\hline Amphisbaena trachura Cope, 1885 & $\mathrm{X}$ & - & - & - & - & - & $\mathrm{X}$ & - & - & - & - \\
\hline Amphisbaena roberti (Gans, 1964) & $\mathrm{X}$ & $\mathrm{X}$ & - & $\mathrm{X}$ & - & - & $\mathrm{X}$ & $\mathrm{X}$ & - & - & - \\
\hline Amphisbaena steindachneri (Strauch, 1881) & $\mathrm{X}$ & - & - & - & - & - & - & - & - & - & - \\
\hline Amphisbaena microcephalum (Wagler, 1824) & - & $\mathrm{X}$ & - & - & - & - & - & - & - & - & - \\
\hline Amphisbaena wuchereri (Peters, 1879) & $\mathrm{X}$ & - & - & - & - & - & $\mathrm{X}$ & - & - & - & - \\
\hline \multicolumn{12}{|l|}{ SQUAMATA - "Lacertilia" } \\
\hline \multicolumn{12}{|l|}{ Anguidae } \\
\hline Diploglossus fasciatus (Gray, 1831) & - & $\mathrm{X}$ & - & - & - & - & - & - & - & - & - \\
\hline Ophiodes fragilis (Raddi, 1820) & $\mathrm{X}$ & $\mathrm{X}$ & $\mathrm{X}$ & $\mathrm{X}$ & - & $\mathrm{X}$ & $\mathrm{X}$ & $\mathrm{X}$ & $\mathrm{X}$ & - & - \\
\hline Ophiodes sp.1 Martins, 1998 & $\mathrm{X}$ & - & - & - & - & - & $\mathrm{X}$ & - & - & - & - \\
\hline Ophiodes sp.2 Martins 1998 & - & - & $\mathrm{X}$ & - & - & - & - & - & - & - & - \\
\hline
\end{tabular}


Tabela 1. Continuação...

\begin{tabular}{|c|c|c|c|c|c|c|c|c|c|c|c|}
\hline Taxon & FES & FOD & FOM & CER & $\begin{array}{c}\text { FOM/ } \\
\text { FES }\end{array}$ & $\begin{array}{l}\text { FOD/ } \\
\text { FOM }\end{array}$ & $\begin{array}{c}\text { CER/ } \\
\text { FES }\end{array}$ & $\begin{array}{l}\text { CER/ } \\
\text { FOD }\end{array}$ & $\begin{array}{l}\text { CER/ } \\
\text { FOM }\end{array}$ & $\mathbf{S P}$ & BR \\
\hline Ophiodes striatus (Spix, 1824) & $\mathrm{X}$ & $\mathrm{X}$ & $\mathrm{X}$ & - & - & - & $\mathrm{X}$ & $\mathrm{X}$ & - & - & - \\
\hline \multicolumn{12}{|l|}{ Leiosauridae } \\
\hline Anisolepis grilli Boulenger, 1891 & $\mathrm{X}$ & $\mathrm{X}$ & - & - & - & - & $\mathrm{X}$ & $\mathrm{X}$ & - & - & - \\
\hline Enyalius iheringii Boulenger, 1885 & $\mathrm{X}$ & $\mathrm{X}$ & $\mathrm{X}$ & - & - & - & $\mathrm{X}$ & $\mathrm{X}$ & $\mathrm{X}$ & - & - \\
\hline Enyalius perditus Jackson, 1978 & $\mathrm{X}$ & $\mathrm{X}$ & - & $\mathrm{X}$ & - & - & $\mathrm{X}$ & - & - & - & - \\
\hline Urostrophus vautieri Duméril \& Bibron, 1837 & $\mathrm{X}$ & $\mathrm{X}$ & $\mathrm{X}$ & - & - & - & $\mathrm{X}$ & $\mathrm{X}$ & - & - & - \\
\hline \multicolumn{12}{|l|}{ Phyllodactylidae } \\
\hline Gymnodactylus darwinii (Gray, 1845) & - & $\mathrm{X}$ & - & - & - & - & - & - & - & - & - \\
\hline \multicolumn{12}{|l|}{ Polychrotidae } \\
\hline Anolis chrysolepis Dumeril \& Bibron, 1837 & $\mathrm{X}$ & - & - & $\mathrm{X}$ & - & - & $\mathrm{X}$ & - & - & - & - \\
\hline Anolis meridionalis Boettger, 1885 & - & $\mathrm{X}$ & - & - & - & - & - & - & - & VU & - \\
\hline Anolis punctatus Daudin, 1802 & - & $\mathrm{X}$ & - & - & - & - & - & - & - & - & - \\
\hline Polychrus acutirostris Spix, 1825 & $\mathrm{X}$ & $\mathrm{X}$ & - & $\mathrm{X}$ & - & - & $\mathrm{X}$ & $\mathrm{X}$ & - & - & - \\
\hline Polychrus marmoratus (Linnaeus, 1758) & - & $\mathrm{X}$ & - & - & - & - & - & - & - & - & - \\
\hline \multicolumn{12}{|l|}{ Tropiduridae } \\
\hline Stenocercus azureus (Müller, 1882) & $\mathrm{X}$ & - & - & - & - & - & $\mathrm{X}$ & - & - & VU & - \\
\hline Tropidurus itambere Rodrigues, 1987 & $\mathrm{X}$ & $\mathrm{X}$ & - & $\mathrm{X}$ & - & - & $\mathrm{X}$ & $\mathrm{X}$ & - & - & - \\
\hline Tropidurus torquatus (Wied, 1820) & $\mathrm{X}$ & $\mathrm{X}$ & - & $\mathrm{X}$ & - & - & - & $\mathrm{X}$ & - & - & - \\
\hline \multicolumn{12}{|l|}{ Scincidae } \\
\hline Mabuya caissara Rebouças-Spieker, 1974* & $\mathrm{X}$ & $\mathrm{X}$ & - & - & - & - & - & - & - & EN & - \\
\hline Mabuya dorsivittata Cope, 1862 & $\mathrm{X}$ & $\mathrm{X}$ & $\mathrm{X}$ & $\mathrm{X}$ & - & - & $\mathrm{X}$ & $\mathrm{X}$ & $\mathrm{X}$ & - & - \\
\hline Mabuya frenata (Cope, 1862) & $\mathrm{X}$ & $\mathrm{X}$ & - & - & - & - & $\mathrm{X}$ & $\mathrm{X}$ & - & - & - \\
\hline Mabuya guaporicola Dunn, 1936 & - & - & - & $\mathrm{X}$ & - & - & - & - & - & VU & - \\
\hline Mabuya macrorhyncha Hoge, 1947* & - & $\mathrm{X}$ & - & - & - & - & - & - & - & - & - \\
\hline Mabuya nigropunctata (Spix, 1825) & $\mathrm{X}$ & - & - & - & - & - & - & - & - & - & - \\
\hline \multicolumn{12}{|l|}{ Gymnophthalmidae } \\
\hline Bachia bresslaui (Amaral, 1935) & - & - & - & $\mathrm{X}$ & - & - & - & - & - & VU & - \\
\hline Cercosaura ocellata Wagler, 1830 & - & $\mathrm{X}$ & $\mathrm{X}$ & - & - & - & $\mathrm{X}$ & - & - & - & - \\
\hline Cercosaura quadrilineata (Boettger, 1876) & - & $\mathrm{X}$ & $\mathrm{X}$ & - & - & - & - & - & - & - & - \\
\hline Cercosaura schreibersii Wiegmann, 1834 & $\mathrm{X}$ & $\mathrm{X}$ & $\mathrm{X}$ & - & - & - & - & $\mathrm{X}$ & $\mathrm{X}$ & VU & - \\
\hline Colobodactylus dalcyanus Vanzolini \& Ramos, 1977 & - & - & $\mathrm{X}$ & - & - & - & - & - & - & - & - \\
\hline Colobodactylus taunayi (Amaral, 1933) & - & $\mathrm{X}$ & $\mathrm{X}$ & - & - & - & $\mathrm{X}$ & $\mathrm{X}$ & - & - & - \\
\hline Colobosaura modesta (Reinhardt \& Lütken, 1862) & $\mathrm{X}$ & - & - & - & - & - & $\mathrm{X}$ & - & - & - & - \\
\hline Ecpleopus gaudichaudii Duméril \& Bibron, 1839 & $\mathrm{X}$ & $\mathrm{X}$ & $\mathrm{X}$ & - & - & - & - & $\mathrm{X}$ & - & - & - \\
\hline Heterodactylus imbricatus Spix, 1825 & $\mathrm{X}$ & $\mathrm{X}$ & - & - & - & - & - & - & - & - & - \\
\hline Micrablepharus atticolus Rodrigues, 1996 & $\mathrm{X}$ & - & - & - & - & - & $\mathrm{X}$ & - & - & VU & - \\
\hline Placosoma champsonotus (Werner, 1910) & $\mathrm{X}$ & $\mathrm{X}$ & $\mathrm{X}$ & - & - & - & - & - & - & - & - \\
\hline Placosoma cordylinum Tschudi, 1847 & - & $\mathrm{X}$ & - & - & - & - & - & - & $\mathrm{X}$ & - & - \\
\hline Placosoma glabellum (Peters, 1870) & - & $\mathrm{X}$ & $\mathrm{X}$ & - & - & $\mathrm{X}$ & - & $\mathrm{X}$ & $\mathrm{X}$ & - & - \\
\hline \multicolumn{12}{|l|}{ Teiidae } \\
\hline Ameiva ameiva (Linnaeus, 1758) & $\mathrm{X}$ & $\mathrm{X}$ & - & $\mathrm{X}$ & - & - & $\mathrm{X}$ & $\mathrm{X}$ & - & - & - \\
\hline Cnemidophorus aff. parecis & $\mathrm{X}$ & - & - & - & - & - & $\mathrm{X}$ & - & - & - & - \\
\hline Cnemidophorus cf. ocellifer & - & - & - & - & - & - & $\mathrm{X}$ & - & - & - & - \\
\hline Kentropyx paulensis Boettger, 1893 & $\mathrm{X}$ & $\mathrm{X}$ & - & $\mathrm{X}$ & - & - & $\mathrm{X}$ & - & - & VU & - \\
\hline Teius oculatus (D’Orbigny \& Bibron, 1837) & - & - & $\mathrm{X}$ & - & - & - & - & - & $\mathrm{X}$ & - & - \\
\hline Tupinambis merianae (Duméril \& Bibron, 1839) & $\mathrm{X}$ & $\mathrm{X}$ & - & $\mathrm{X}$ & - & - & $\mathrm{X}$ & $\mathrm{X}$ & $\mathrm{X}$ & - & - \\
\hline Tupinambis palustris Manzani \& Abe, 2002 & $\mathrm{X}$ & $\mathrm{X}$ & - & $\mathrm{X}$ & - & - & - & - & - & - & - \\
\hline \multicolumn{12}{|l|}{ SQUAMATA - Serpentes } \\
\hline \multicolumn{12}{|l|}{ Anomalepididae } \\
\hline Liotyphlops beui (Amaral, 1924) & $\mathrm{X}$ & $\mathrm{X}$ & - & - & - & - & - & - & - & - & - \\
\hline
\end{tabular}


Tabela 1. Continuação...

\begin{tabular}{|c|c|c|c|c|c|c|c|c|c|c|c|}
\hline Taxon & FES & FOD & FOM & CER & $\begin{array}{l}\text { FOM/ } \\
\text { FES }\end{array}$ & $\begin{array}{l}\text { FOD/ } \\
\text { FOM }\end{array}$ & $\begin{array}{l}\text { CER/ } \\
\text { FES }\end{array}$ & $\begin{array}{l}\text { CER/ } \\
\text { FOD }\end{array}$ & $\begin{array}{l}\text { CER/ } \\
\text { FOM }\end{array}$ & $\mathbf{S P}$ & $\mathbf{B R}$ \\
\hline Liotyphlops caissara Centeno, Sawaya \& Germano, 2010* & - & $\mathrm{X}$ & - & - & - & - & - & - & - & - & - \\
\hline Liotyphlops schubarti Vanzolini, 1948* & - & $\mathrm{X}$ & - & - & - & - & - & - & - & VU & - \\
\hline Liotyphlops ternetzii (Boulenger, 1896) & $\mathrm{X}$ & - & - & $\mathrm{X}$ & - & - & - & - & - & - & - \\
\hline \multicolumn{12}{|l|}{ Leptotyphlopidae } \\
\hline Tricheilostoma koppesi (Amaral, 1955) & $\mathrm{X}$ & $\mathrm{X}$ & - & $\mathrm{X}$ & - & - & - & - & - & - & - \\
\hline \multicolumn{12}{|l|}{ Typhlopidae } \\
\hline Typhlops brongersmianus Vanzolini, 1976 & $\mathrm{X}$ & $\mathrm{X}$ & - & - & - & - & - & - & - & - & - \\
\hline \multicolumn{12}{|l|}{ Tropidophiidae } \\
\hline Tropidophis paucisquamis (Müller, 1901) & - & $\mathrm{X}$ & $\mathrm{X}$ & - & - & $\mathrm{X}$ & - & $\mathrm{X}$ & - & - & - \\
\hline \multicolumn{12}{|l|}{ Boidae } \\
\hline Boa constrictor Linnaeus, 1758 & $\mathrm{X}$ & $\mathrm{X}$ & - & $\mathrm{X}$ & - & - & $\mathrm{X}$ & $\mathrm{X}$ & $\mathrm{X}$ & - & - \\
\hline Corallus cropanii (Hoge, 1953)* & - & $\mathrm{X}$ & - & - & - & - & - & - & - & EN & $\mathrm{CR}$ \\
\hline Corallus hortulanus (Linnaeus, 1758) & - & $X$ & - & - & - & - & - & - & - & - & - \\
\hline Epicrates cenchria (Linnaeus, 1758) & - & - & - & - & - & - & $\mathrm{X}$ & - & - & - & - \\
\hline Epicrates crassus Cope, 1862 & $\mathrm{X}$ & $\mathrm{X}$ & $\mathrm{X}$ & $\mathrm{X}$ & - & - & $\mathrm{X}$ & $\mathrm{X}$ & - & - & - \\
\hline Eunectes murinus (Linnaeus, 1758) & $\mathrm{X}$ & - & - & $\mathrm{X}$ & - & - & $\mathrm{X}$ & - & - & - & - \\
\hline \multicolumn{12}{|l|}{ Colubridae } \\
\hline Chironius bicarinatus (Wied, 1820) & $\mathrm{X}$ & $\mathrm{X}$ & $\mathrm{X}$ & $\mathrm{X}$ & - & $\mathrm{X}$ & $\mathrm{X}$ & $\mathrm{X}$ & $\mathrm{X}$ & - & - \\
\hline Chironius exoletus (Linnaeus, 1758) & $\mathrm{X}$ & $\mathrm{X}$ & $\mathrm{X}$ & $\mathrm{X}$ & - & $\mathrm{X}$ & $\mathrm{X}$ & $\mathrm{X}$ & $\mathrm{X}$ & - & - \\
\hline Chironius flavolineatus (Boettger, 1885) & $\mathrm{X}$ & $\mathrm{X}$ & - & $\mathrm{X}$ & - & - & $\mathrm{X}$ & $\mathrm{X}$ & $\mathrm{X}$ & - & - \\
\hline Chironius foveatus Bailey, 1955 & - & $\mathrm{X}$ & - & - & - & - & - & $\mathrm{X}$ & - & - & - \\
\hline Chironius fuscus (Linnaeus, 1758) & $\mathrm{X}$ & $\mathrm{X}$ & - & - & - & - & - & - & - & - & - \\
\hline Chironius laevicollis (Wied, 1824) & $\mathrm{X}$ & $\mathrm{X}$ & - & - & - & - & $\mathrm{X}$ & $\mathrm{X}$ & - & - & - \\
\hline Chironius quadricarinatus (Boie, 1827) & $\mathrm{X}$ & $\mathrm{X}$ & - & $\mathrm{X}$ & - & - & $\mathrm{X}$ & $\mathrm{X}$ & - & - & - \\
\hline Drymarchon corais (Boie, 1827) & $\mathrm{X}$ & - & - & $\mathrm{X}$ & - & - & $\mathrm{X}$ & - & - & - & - \\
\hline Drymoluber brazili (Gomes, 1918) & $\mathrm{X}$ & - & - & $\mathrm{X}$ & - & - & $\mathrm{X}$ & - & - & - & - \\
\hline Leptophis ahaetulla (Linnaeus, 1758) & $\mathrm{X}$ & - & - & - & - & - & $\mathrm{X}$ & - & - & - & - \\
\hline Mastigodryas bifossatus (Raddi, 1820) & $\mathrm{X}$ & $\mathrm{X}$ & - & $\mathrm{X}$ & - & - & $\mathrm{X}$ & $\mathrm{X}$ & - & - & - \\
\hline Pseustes sulphureus (Wagler, 1824) & - & $\mathrm{X}$ & - & - & - & - & - & - & - & - & - \\
\hline Simophis rhinostoma (Schlegel, 1837) & $\mathrm{X}$ & $\mathrm{X}$ & - & $\mathrm{X}$ & - & - & $\mathrm{X}$ & $\mathrm{X}$ & $\mathrm{X}$ & - & - \\
\hline Spilotes pullatus (Linnaeus, 1758) & $\mathrm{X}$ & $X$ & - & $\mathrm{X}$ & - & - & $\mathrm{X}$ & - & $\mathrm{X}$ & - & - \\
\hline Tantilla melanocephala (Linnaeus, 1758) & $\mathrm{X}$ & $\mathrm{X}$ & $\mathrm{X}$ & $\mathrm{X}$ & - & - & - & $\mathrm{X}$ & - & - & - \\
\hline \multicolumn{12}{|l|}{ Dipsadidae } \\
\hline Apostolepis assimilis (Reinhardt, 1861) & $\mathrm{X}$ & $\mathrm{X}$ & $\mathrm{X}$ & - & $\mathrm{X}$ & - & $\mathrm{X}$ & $\mathrm{X}$ & - & - & - \\
\hline Apostolepis dimidiata (Jan, 1862) & $\mathrm{X}$ & $\mathrm{X}$ & $\mathrm{X}$ & $\mathrm{X}$ & - & - & $\mathrm{X}$ & $\mathrm{X}$ & $\mathrm{X}$ & - & - \\
\hline $\begin{array}{l}\text { Apostolepis flavotorquata (Duméril, Bibron \& Duméril, } \\
\text { 1854) }\end{array}$ & $\mathrm{X}$ & - & - & - & - & - & - & - & - & - & - \\
\hline Apostolepis goiasensis Prado, 1942 & $\mathrm{X}$ & - & - & - & - & - & - & - & - & - & - \\
\hline $\begin{array}{l}\text { Atractus francoi Passos, Fernandes, Bérnils \& Moura- } \\
\text { Leite, } 2010\end{array}$ & $\mathrm{X}$ & $\mathrm{X}$ & - & - & - & - & - & - & - & - & - \\
\hline Atractus pantostictus Fernandes \& Puorto, 1993 & $\mathrm{X}$ & $\mathrm{X}$ & - & $\mathrm{X}$ & - & - & $\mathrm{X}$ & - & - & - & - \\
\hline Atractus reticulatus (Boulenger, 1885) & - & $\mathrm{X}$ & $\mathrm{X}$ & - & - & - & $\mathrm{X}$ & - & - & - & - \\
\hline Atractus serranus Amaral, 1930* & - & $\mathrm{X}$ & - & $\mathrm{X}$ & - & - & - & $\mathrm{X}$ & - & - & - \\
\hline Atractus trihedrurus Amaral, 1926 & - & $\mathrm{X}$ & - & - & - & - & - & $\mathrm{X}$ & - & - & - \\
\hline Atractus zebrinus (Jan, 1862) & $\mathrm{X}$ & $X$ & $X$ & - & - & $\mathrm{X}$ & - & - & - & - & - \\
\hline Boiruna maculata (Boulenger, 1896) & $\mathrm{X}$ & $\mathrm{X}$ & - & $\mathrm{X}$ & - & - & $X$ & $\mathrm{X}$ & $\mathrm{X}$ & - & - \\
\hline Caaeteboia amarali (Wettstein, 1930) & - & $\mathrm{X}$ & - & - & - & - & - & - & - & VU & - \\
\hline Clelia aff. rustica & $\mathrm{X}$ & - & - & - & - & - & - & - & - & - & - \\
\hline Clelia plumbea (Wied, 1820) & $\mathrm{X}$ & $\mathrm{X}$ & - & $\mathrm{X}$ & - & - & $\mathrm{X}$ & $\mathrm{X}$ & - & - & - \\
\hline Clelia rustica (Cope, 1878) & - & $\mathrm{X}$ & - & - & - & - & $\mathrm{X}$ & - & - & VU & - \\
\hline Dipsas albifrons (Sauvage, 1884) & $\mathrm{X}$ & $\mathrm{X}$ & - & - & - & $\mathrm{X}$ & - & - & - & - & $\mathrm{CR}$ \\
\hline Dipsas alternans (Fischer, 1885) & $\mathrm{X}$ & $\mathrm{X}$ & $\mathrm{X}$ & - & - & $\mathrm{X}$ & - & - & - & - & - \\
\hline
\end{tabular}


Tabela 1. Continuação...

\begin{tabular}{|c|c|c|c|c|c|c|c|c|c|c|c|}
\hline Taxon & FES & FOD & FOM & CER & $\begin{array}{c}\text { FOM/ } \\
\text { FES }\end{array}$ & $\begin{array}{l}\text { FOD/ } \\
\text { FOM }\end{array}$ & $\begin{array}{l}\text { CER/ } \\
\text { FES }\end{array}$ & $\begin{array}{l}\text { CER/ } \\
\text { FOD }\end{array}$ & $\begin{array}{l}\text { CER/ } \\
\text { FOM }\end{array}$ & $\mathbf{S P}$ & BR \\
\hline Dipsas indica (Laurenti, 1768) & $\mathrm{X}$ & $\mathrm{X}$ & - & $\mathrm{X}$ & - & - & $\mathrm{X}$ & - & $\mathrm{X}$ & - & - \\
\hline Dipsas neivai Amaral, 1926 & - & $\mathrm{X}$ & - & - & - & - & - & $\mathrm{X}$ & - & - & - \\
\hline Ditaxodon taeniatus (Peters in Hensel, 1868) & $\mathrm{X}$ & - & - & - & - & - & - & - & - & VU & - \\
\hline Echinanthera amoena (Jan, 1863) & - & $\mathrm{X}$ & - & - & - & - & - & - & - & - & - \\
\hline Echinanthera cephalostriata Di-Bernardo, 1996 & $\mathrm{X}$ & $\mathrm{X}$ & $\mathrm{X}$ & $\mathrm{X}$ & - & $\mathrm{X}$ & - & $\mathrm{X}$ & - & - & - \\
\hline Echinanthera cyanopleura (Cope, 1885) & - & $\mathrm{X}$ & - & - & - & - & - & $\mathrm{X}$ & - & - & - \\
\hline Echinanthera melanostigma (Wagler, 1824) & $\mathrm{X}$ & $\mathrm{X}$ & - & - & - & - & - & - & - & - & - \\
\hline Echinanthera undulata (Wied, 1824) & $\mathrm{X}$ & $\mathrm{X}$ & $\mathrm{X}$ & $\mathrm{X}$ & - & $\mathrm{X}$ & $\mathrm{X}$ & $\mathrm{X}$ & - & - & - \\
\hline Elapomorphus quinquelineatus (Raddi, 1820) & - & $\mathrm{X}$ & - & $\mathrm{X}$ & - & - & - & - & - & - & - \\
\hline Erythrolamprus aesculapii (Linnaeus, 1766) & $\mathrm{X}$ & $\mathrm{X}$ & $\mathrm{X}$ & $\mathrm{X}$ & - & - & $\mathrm{X}$ & $\mathrm{X}$ & - & - & - \\
\hline Erythrolamprus almadensis (Wagler, 1824) & $\mathrm{X}$ & $\mathrm{X}$ & - & $\mathrm{X}$ & - & - & $\mathrm{X}$ & $\mathrm{X}$ & - & - & - \\
\hline Erythrolamprus atraventer (Dixon \& Thomas, 1985) & $\mathrm{X}$ & $\mathrm{X}$ & - & - & - & - & - & - & - & - & - \\
\hline Erythrolamprus frenatus (Werner, 1909) & $\mathrm{X}$ & $\mathrm{X}$ & - & $\mathrm{X}$ & - & - & $\mathrm{X}$ & - & - & - & - \\
\hline Erythrolamprus jaegeri (Günther, 1858) & $\mathrm{X}$ & $\mathrm{X}$ & $\mathrm{X}$ & $\mathrm{X}$ & - & - & $\mathrm{X}$ & $\mathrm{X}$ & $\mathrm{X}$ & - & - \\
\hline Erythrolamprus miliaris (Linnaeus, 1758) & $\mathrm{X}$ & $\mathrm{X}$ & $\mathrm{X}$ & $\mathrm{X}$ & - & $\mathrm{X}$ & $\mathrm{X}$ & $\mathrm{X}$ & $\mathrm{X}$ & - & - \\
\hline Erythrolamprus poecilogyrus (Wied, 1825) & $\mathrm{X}$ & $\mathrm{X}$ & $\mathrm{X}$ & $\mathrm{X}$ & $\mathrm{X}$ & - & $\mathrm{X}$ & $\mathrm{X}$ & $\mathrm{X}$ & - & - \\
\hline Erythrolamprus reginae (Linnaeus, 1758) & $\mathrm{X}$ & $\mathrm{X}$ & - & $\mathrm{X}$ & - & - & $\mathrm{X}$ & - & - & - & - \\
\hline Erythrolamprus typhlus (Linnaeus, 1758) & $\mathrm{X}$ & $\mathrm{X}$ & - & $\mathrm{X}$ & - & - & $\mathrm{X}$ & $\mathrm{X}$ & $\mathrm{X}$ & - & - \\
\hline Gomesophis brasiliensis (Gomes, 1918) & $\mathrm{X}$ & $\mathrm{X}$ & - & $\mathrm{X}$ & - & - & - & - & - & - & - \\
\hline Helicops carinicaudus (Wied, 1825) & - & $\mathrm{X}$ & - & - & - & - & - & - & - & - & - \\
\hline Helicops gomesi Amaral, 1921 & $\mathrm{X}$ & $\mathrm{X}$ & - & $\mathrm{X}$ & - & - & $\mathrm{X}$ & - & - & - & - \\
\hline Helicops infrataeniatus (Jan, 1865) & $\mathrm{X}$ & - & - & $\mathrm{X}$ & - & - & $\mathrm{X}$ & - & - & - & - \\
\hline Helicops modestus Günther, 1861 & $\mathrm{X}$ & - & - & $\mathrm{X}$ & - & - & $\mathrm{X}$ & $\mathrm{X}$ & - & - & - \\
\hline Hydrodynastes bicinctus (Herrmann, 1804) & $\mathrm{X}$ & - & - & $\mathrm{X}$ & - & - & $\mathrm{X}$ & - & - & - & - \\
\hline Hydrodynastes gigas (Duméril, Bibron \& Duméril, 1854) & $\mathrm{X}$ & $\mathrm{X}$ & - & $\mathrm{X}$ & - & - & $\mathrm{X}$ & - & - & - & - \\
\hline Imantodes cenchoa (Linnaeus, 1758) & $\mathrm{X}$ & $\mathrm{X}$ & - & $\mathrm{X}$ & - & - & $\mathrm{X}$ & $\mathrm{X}$ & - & - & - \\
\hline Leptodeira annulata (Linnaeus, 1758) & $\mathrm{X}$ & $\mathrm{X}$ & - & $\mathrm{X}$ & - & - & $\mathrm{X}$ & $\mathrm{X}$ & - & - & - \\
\hline Lygophis flavifrenatus Cope, 1862 & - & $\mathrm{X}$ & - & - & - & - & - & - & - & - & - \\
\hline Lygophis meridionalis (Schenkel, 1901) & $\mathrm{X}$ & $\mathrm{X}$ & - & $\mathrm{X}$ & - & - & $\mathrm{X}$ & $\mathrm{X}$ & - & - & - \\
\hline Mussurana montana (Franco, Marques \& Puorto, 1997) & $\mathrm{X}$ & - & $\mathrm{X}$ & - & - & - & - & - & - & VU & - \\
\hline Mussurana quimi (Franco, Marques \& Puorto, 1997) & $\mathrm{X}$ & $\mathrm{X}$ & - & $\mathrm{X}$ & - & - & $\mathrm{X}$ & $\mathrm{X}$ & - & - & - \\
\hline Oxyrhopus clathratus Duméril, Bibron \& Duméril, 1854 & $\mathrm{X}$ & $\mathrm{X}$ & $\mathrm{X}$ & - & $\mathrm{X}$ & - & - & $\mathrm{X}$ & $\mathrm{X}$ & - & - \\
\hline Oxyrhopus guibei Hoge \& Romano, 1978 & $\mathrm{X}$ & $\mathrm{X}$ & $\mathrm{X}$ & $\mathrm{X}$ & - & - & $\mathrm{X}$ & $\mathrm{X}$ & - & - & - \\
\hline Oxyrhopus petola (Linnaeus, 1758) & $\mathrm{X}$ & $\mathrm{X}$ & - & $\mathrm{X}$ & - & - & $\mathrm{X}$ & - & - & - & - \\
\hline Oxyrhopus rhombifer Duméril, Bibron \& Duméril, 1854 & - & $\mathrm{X}$ & - & - & - & - & $\mathrm{X}$ & - & $\mathrm{X}$ & - & - \\
\hline Phalotris lativittatus Ferrarezzi, 1994* & $\mathrm{X}$ & - & - & $\mathrm{X}$ & - & - & $\mathrm{X}$ & - & - & - & - \\
\hline $\begin{array}{l}\text { Phalotris matogrossensis Lema, D’Agostini \& Cappellari, } \\
2005\end{array}$ & $\mathrm{X}$ & - & - & - & - & - & - & - & - & - & - \\
\hline Phalotris mertensi (Hoge, 1955) & $\mathrm{X}$ & $\mathrm{X}$ & - & $\mathrm{X}$ & - & - & $\mathrm{X}$ & $\mathrm{X}$ & - & - & - \\
\hline Phalotris multipunctatus Puorto \& Ferrarezzi, 1994 & - & - & - & $\mathrm{X}$ & - & - & - & - & - & $\mathrm{EN}$ & - \\
\hline Phalotris nasutus (Gomes, 1915) & $\mathrm{X}$ & - & - & - & - & - & $\mathrm{X}$ & - & - & $\mathrm{EN}$ & - \\
\hline Phalotris reticulatus (Peters, 1860) & - & $\mathrm{X}$ & $\mathrm{X}$ & - & - & - & - & - & - & - & - \\
\hline Philodryas aestiva Duméril, Bibron \& Duméril, 1854) & $\mathrm{X}$ & $\mathrm{X}$ & - & - & - & - & $\mathrm{X}$ & $\mathrm{X}$ & - & - & - \\
\hline Philodryas agassizii (Jan, 1863) & - & - & - & $\mathrm{X}$ & - & - & - & - & - & VU & - \\
\hline Philodryas arnaldoi (Amaral, 1932) & - & - & - & - & - & - & $\mathrm{X}$ & - & - & EN & - \\
\hline Philodryas livida (Amaral, 1923) & $\mathrm{X}$ & - & - & $\mathrm{X}$ & - & - & $\mathrm{X}$ & - & - & VU & - \\
\hline Philodryas mattogrossensis Koslowsky, 1898 & $\mathrm{X}$ & - & - & $\mathrm{X}$ & - & - & $\mathrm{X}$ & - & - & - & - \\
\hline Philodryas nattereri Steindachner, 1870 & $\mathrm{X}$ & - & - & - & - & - & $\mathrm{X}$ & - & - & - & - \\
\hline Philodryas olfersii (Lichtenstein, 1823) & $\mathrm{X}$ & $\mathrm{X}$ & - & $\mathrm{X}$ & - & - & $\mathrm{X}$ & $\mathrm{X}$ & $\mathrm{X}$ & - & - \\
\hline Philodryas patagoniensis (Girard, 1857) & $\mathrm{X}$ & $\mathrm{X}$ & $\mathrm{X}$ & $\mathrm{X}$ & - & - & $\mathrm{X}$ & - & - & - & - \\
\hline Phimophis guerini (Duméril, Bibron \& Duméril, 1854) & $\mathrm{X}$ & - & - & $\mathrm{X}$ & - & - & $\mathrm{X}$ & - & - & - & - \\
\hline Pseudoboa haasi (Boettger, 1905) & $\mathrm{X}$ & - & $\mathrm{X}$ & - & - & $\mathrm{X}$ & - & - & - & - & - \\
\hline
\end{tabular}


Tabela 1. Continuação...

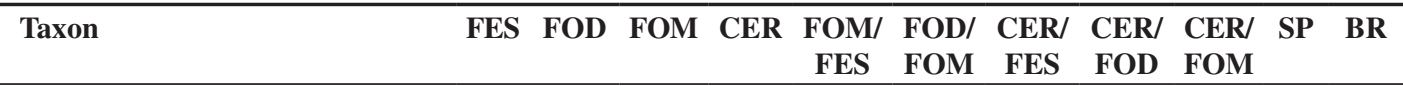

Pseudoboa nigra (Duméril, Bibron \& Duméril, 1854)

Pseudoboa serrana Morato, Moura-Leite, Prudente \& Bérnils, 1995

Psomophis joberti (Sauvage, 1884)

Rhachidelus brazili Boulenger, 1908

Sibynomorphus mikanii (Schlegel, 1837)

Sibynomorphus neuwiedi (Ihering, 1911)

Sibynomorphus ventrimaculatus (Boulenger, 1885)

Siphlophis longicaudatus (Andersson, 1907)

Siphlophis pulcher (Raddi, 1820)

Sordellina punctata (Peters, 1880)

Taeniophallus affinis (Günther, 1858)

Taeniophallus bilineatus (Fischer, 1885)

Taeniophallus occipitalis (Jan, 1863)

Taeniophallus persimilis (Cope, 1869)

Thamnodynastes hypoconia (Cope, 1860)

$\begin{array}{lllllllllll}X & - & - & X & - & - & X & - & - & - & - \\ - & X & - & - & - & X & - & - & - & - & -\end{array}$

Thamnodynastes longicaudus Franco, Ferreira, Marques \& Sazima, 2003

Thamnodynastes nattereri (Mikan, 1828)

Thamnodynastes rutilus (Prado, 1942)

Thamnodynastes strigatus (Günther, 1858)

Tomodon dorsatus Duméril, Bibron \& Duméril, 1854

Tropidodryas serra (Schlegel, 1837)

Tropidodryas striaticeps (Cope, 1869)

Uromacerina ricardinii (Peracca, 1897)

Xenodon histricus (Jan, 1863)

Xenodon merremii (Wagler, 1824)

Xenodon nattereri (Steindachner, 1867)

Xenodon neuwiedii Günther, 1863

Xenopholis scalaris (Wucherer, 1861)

Xenopholis undulatus (Jensen, 1900)

Elapidae

Micrurus corallinus (Merrem, 1820)

Micrurus decoratus (Jan, 1858)

Micrurus frontalis (Duméril, Bibron \& Duméril, 1854)

Micrurus lemniscatus (Linnaeus, 1758)

$\begin{array}{llll}X & - & - & - \\ X & X & - & X \\ X & X & - & X \\ - & X & - & - \\ - & - & X & - \\ - & X & - & - \\ - & X & - & - \\ X & X & - & - \\ - & X & - & - \\ - & X & - & - \\ X & X & X & - \\ X & X & X & X \\ X & X & - & X \\ X & X & X & -\end{array}$

Viperidae

Bothrops jararacussu Lacerda, 1884

Bothrops moojeni Hoge, 1966

Bothropoides alcatraz (Marques, Martins \& Sazima, 2002)*

Bothropoides insularis (Amaral, 1921)*

Bothropoides jararaca (Wied, 1824)

Bothropoides mattogrossensis (Amaral, 1925)

Bothropoides neuwiedi (Wagler, 1824)

Bothropoides pauloensis (Amaral, 1925)

Rhinocerophis alternatus (Duméril, Bibron \& Duméril, 1854)

Rhinocerophis cotiara (Gomes, 1913)

Rhinocerophis fonsecai (Hoge \& Belluomini, 1959)

Rhinocerophis itapetiningae (Boulenger, 1907)

Crotalus durissus Linnaeus, 1758

$$
\begin{array}{lllllllllll}
- & \mathrm{X} & - & - & - & - & - & - & - & - & - \\
\mathrm{X} & - & - & - & - & - & \mathrm{X} & - & - & - & - \\
- & \mathrm{X} & \mathrm{X} & \mathrm{X} & - & - & \mathrm{X} & \mathrm{X} & - & - & - \\
\mathrm{X} & \mathrm{X} & \mathrm{X} & - & - & - & - & \mathrm{X} & - & - & - \\
- & \mathrm{X} & - & - & - & - & - & - & - & - & - \\
- & \mathrm{X} & - & - & - & - & - & - & - & - & - \\
- & \mathrm{X} & - & - & - & - & - & - & - & - & - \\
- & - & - & \mathrm{X} & - & - & - & - & - & - & - \\
\mathrm{X} & \mathrm{X} & \mathrm{X} & \mathrm{X} & - & - & \mathrm{X} & \mathrm{X} & - & - & - \\
\mathrm{X} & \mathrm{X} & - & \mathrm{X} & - & - & \mathrm{X} & - & - & \mathrm{VU} & - \\
\mathrm{X} & \mathrm{X} & \mathrm{X} & \mathrm{X} & - & - & \mathrm{X} & \mathrm{X} & - & - & - \\
- & \mathrm{X} & - & - & - & - & - & - & - & - & - \\
\mathrm{X} & - & - & - & - & - & - & - & - & \mathrm{VU} & - \\
& & & & & & & & & & - \\
\mathrm{X} & \mathrm{X} & - & \mathrm{X} & - & - & \mathrm{X} & \mathrm{X} & - & - & - \\
\mathrm{X} & \mathrm{X} & - & \mathrm{X} & - & \mathrm{X} & - & - & - & - & - \\
\mathrm{X} & \mathrm{X} & \mathrm{X} & \mathrm{X} & \mathrm{X} & - & \mathrm{X} & \mathrm{X} & \mathrm{X} & - & - \\
\mathrm{X} & \mathrm{X} & - & \mathrm{X} & - & - & \mathrm{X} & \mathrm{X} & - & - & -
\end{array}
$$

\begin{tabular}{ccccccccccc} 
X & $X$ & $X$ & - & - & - & $X$ & - & - & - & - \\
$X$ & $X$ & - & $X$ & - & - & $X$ & $X$ & - & - & - \\
- & $X$ & - & - & - & - & - & - & - & $C R$ & CR \\
- & $X$ & - & - & - & - & - & - & - & CR & CR \\
$X$ & $X$ & $X$ & $X$ & - & $X$ & $X$ & $X$ & - & - & - \\
$X$ & - & - & $X$ & - & - & - & - & - & - & - \\
- & $X$ & $X$ & - & - & - & - & $X$ & - & - & - \\
$X$ & $X$ & - & - & - & - & $X$ & $X$ & - & - & - \\
$X$ & - & - & - & - & - & $X$ & $X$ & - & - & - \\
& & & & & & & & & & \\
$X$ & - & $X$ & - & - & - & - & - & - & $V U$ & - \\
$X$ & $X$ & $X$ & $X$ & - & $X$ & - & $X$ & - & $V U$ & - \\
$X$ & $X$ & $X$ & $X$ & - & - & $X$ & $X$ & $X$ & $V U$ & - \\
$X$ & $X$ & - & $X$ & - & - & $X$ & $X$ & - & - & - \\
141 & 150 & 55 & 90 & 4 & 16 & 108 & 79 & 29 & 32 & 9 \\
\hline
\end{tabular}




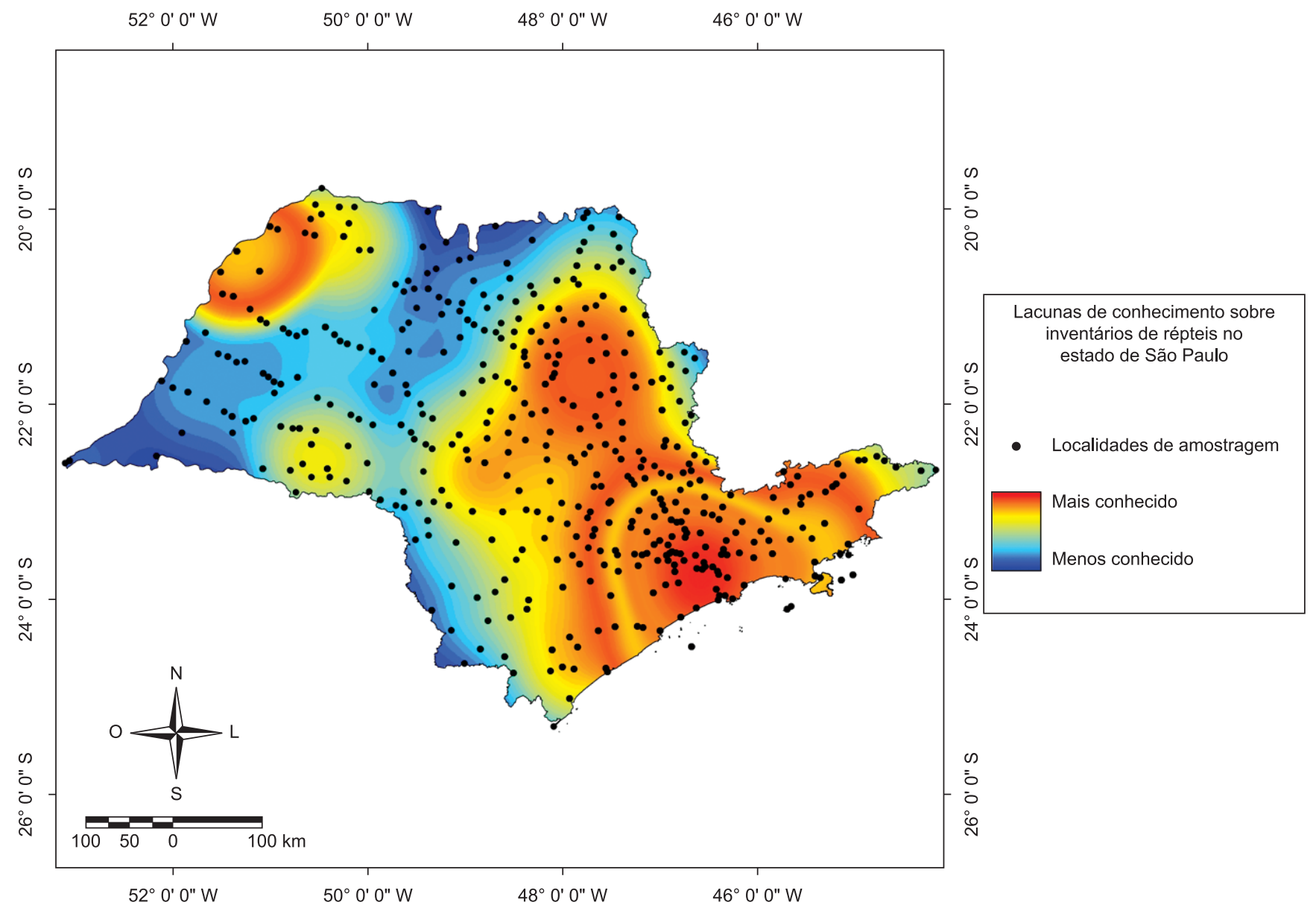

Figura 2. Distribuição dos registros de répteis no Estado de São Paulo (pontos escuros) e zonas de elevado e reduzido conhecimento ou amostragens de répteis em coleções científicas, geradas pela interpolação circular neighbourhood no programa Diva-Gis.

Figure 2. Distribution of reptilian species records in the state of São Paulo (dark dots) with areas of higher and lower levels of reptile sampling in collections, generated through the program Diva-Gis by neighbourhood circular interpolation.

No entanto, há comparações ainda mais ilustrativas. Listas preliminares da fauna de répteis do Estado do Tocantins (Dornas 2009, C. Nogueira dados não publicados) no centro-norte do Brasil, com área similar ao Estado de São Paulo, indicam apenas 144 espécies de répteis (nove quelônios, três crocodilianos, sete anfisbenas, 43 lagartos e 82 serpentes), ou seja, uma riqueza bem menor do que a registrada em São Paulo. Embora o Estado do Tocantins seja dominado por vegetação de Cerrado em bom estado de preservação, o inventário de sua biodiversidade é muito mais recente. A presença de paisagens ainda bem conservadas no estado propiciou levantamentos exaustivos que revelaram endemismos restritos associados a tipos particulares de solos e de paisagens, que passariam despercebidos após perturbação antrópica (Castro-Mello 2003, Colli et al. 2003, Ribeiro et al. 2009, Rodrigues et al. 2007, 2008). Como comentamos acima, é provável que casos similares ocorram em São Paulo, mas a identificação destes locais representa hoje uma tarefa muito difícil frente à descaracterização generalizada das paisagens originais do estado. Diante das perdas de hábitat no estado de São Paulo, muito da diversidade pretérita só pode ser resgatada hoje em dia a partir dos exemplares depositados nas coleções zoológicas.

Pode-se concluir que, quando se trata de conhecimento faunístico acumulado, São Paulo se beneficia de uma situação privilegiada em relação aos demais estados, que ainda se encontram, em sua maioria, em um estágio incipiente de levantamento e documentação da sua biodiversidade. Deve-se dizer, contudo, que esta vantagem resulta apenas de uma precedência histórica na criação de instituições científicas e de núcleos de pesquisa voltados para o estudo da biodiversidade. Felizmente, os esforços de amostragem conduzidos pelos pesquisadores e as suas instituições científicas ao longo do século XX resultaram na construção e preservação de coleçõestestemunho de uma parte significativa dos hábitats destruídos pelo avanço da fronteira agrícola no estado. Para muitas áreas, contudo, não temos sequer informação sobre a fauna original que ali vivia. São por estas razões que, ao mesmo tempo em que é preciso consolidar as informações disponíveis para São Paulo, é também indispensável investir em outras regiões, para que a própria diversidade no estado seja melhor compreendida e contextualizada. É crucial utilizar, de modo adequado, os dados disponíveis em São Paulo para o direcionamento de políticas públicas voltadas à proteção de espécies e conservação da biodiversidade, uma vez que poucas regiões do Brasil aliam o grau de conhecimento faunístico acumulado ao estado crítico de sua cobertura original de vegetação.

\section{Principais avanços relacionados ao programa biota}

A herpetofauna brasileira conta hoje com 721 espécies reconhecidas de lagartos, cobras, anfisbenas, jacarés e tartarugas (Bérnils 2010). No contexto brasileiro, o Estado de São Paulo é uma região relativamente bem estudada, contando com algumas das maiores e mais representativas coleções científicas do país. Apesar disso este patrimônio ainda se encontra em fase inicial de organização para 
que possamos contar com um quadro de informações funcional sobre a diversidade e distribuição dos répteis do estado. Em 1998, foram registradas 186 espécies de répteis no estado (Marques et al. 1998). Uma revisão mais recente, realizada dez anos depois (RossaFeres et al. 2008), sugere a ocorrência de cerca de 200 espécies no estado. Outra revisão recente (Marques et al. 2009a) lista 215 espécies conhecidas para o estado, incluindo sete subespécies e uma espécie exótica (Hemidactylus mabouia). No presente trabalho não incluímos subespécies ou espécies exóticas. Em ambos os trabalhos, foram incluídas espécies ainda não descritas, mas de reconhecida validade taxonômica. Assim, a riqueza de espécies que registramos no presente trabalho $(\mathrm{n}=212)$ é semelhante àquela registrada por Marques et al. (2009a), com exceção de Leposternon infraorbitale para a qual não encontramos espécimes-testemunho nem registros na literatura. Dessa forma, mesmo considerando a ampla distribuição desta espécie no Brasil (Perez \& Ribeiro 2008), a mesma não foi incluída na nossa lista. Os números apresentados aqui e ilustrados pela curva de acumulação de espécies (Figura 1) demonstram claramente que tanto os esforços adicionais visando uma maior amostragem no campo como a intensificação dos estudos taxonômicos revisivos deverão conduzir a um aumento no número de espécies de répteis registradas para o estado.

\section{Principais acervos e grupos de pesquisa no Estado de São Paulo}

- Museu de Zoologia da Universidade de São Paulo (MZUSP) É a maior coleção de répteis da América Latina, representando também uma das maiores coleções mundiais de répteis neotropicais. Conta com acervos expressivos de répteis de outros países da América do Sul, como Argentina, Chile, Colômbia, Ecuador e Guyana. Atualmente possui 120.532 exemplares, incluindo todos os grupos de répteis (100.186 lagartos, 17.983 serpentes, 1.967 tartarugas e 396 crocodilianos). Possui boa infra-estrutura e conta com serviço especializado para a sua manutenção. Está parcialmente informatizada.

- Coleção Herpetológica Alphonse Richard Hoge do Instituto Butantan (IBSP) - Localizada há mais de um século no Instituto Butantan, era a maior e mais importante coleção de serpentes neotropicais, mas foi quase completamente destruída durante um incêndio ocorrido no dia 15 de maio de 2010. Uma fração ainda não contabilizada de exemplares foi resgatada do local após o incêndio. A coleção contava com mais de 70.000 exemplares de serpentes provenientes de inúmeras localidades, principalmente do Brasil e de outros países da América Latina, além de algumas centenas de lagartos e anfisbenas brasileiros. $\mathrm{O}$ acervo contava com praticamente toda a base de dados informatizada, embora não completamente conferida.

- Museu de História Natural “Adão José Cardoso", Universidade Estadual de Campinas (ZUEC) -É uma coleção de pequeno porte, com 3.518 exemplares. Apresenta boa infra-estrutura, contando com funcionários especializados para a sua manutenção. Está totalmente informatizada, mas ainda não completamente conferida.

Os principais grupos de pesquisa de répteis no Estado, abaixo relacionados, são apresentados por Instituição de Ensino e Pesquisa. A recente e expressiva expansão no número de grupos de pesquisa explica o aumento significativo sobre o conhecimento dos répteis do Estado ocorrido nestes últimos anos:

- Instituto Butantan/São Paulo: Laboratório de Ecologia e Evolução (Otávio A. V. Marques, Hebert Ferrarezzi, Maria José J. Silva, Nancy Oguiura e Selma M. Almeida-Santos) - história natural, conservação, reprodução, sistemática, citogenética, toxinologia e evolução.

- Instituto Butantan/São Paulo: Laboratório de Herpetologia (Francisco L. Franco, Paulo Passos, Maria de Fátima Furtado, Maria da Graça Salomão e Radenka Batistick) - citogenética, taxonomia, sistemática e toxinologia.
- Instituto Butantan/São Paulo: Laboratório de Biologia Celular (Carlos A. G. S. Jared e Marta M. Antoniazzi) - morfologia.

- Instituto Butantan/São Paulo: Museu Biológico (Giuseppe Puorto e Hana Suzuki) - taxonomia.

- Instituto Florestal/São Paulo: (Cybele O. Araújo) - composição e distribuição.

- UNESP/Campus de Rio Claro: Departamento de Zoologia (Augusto S. Abe, Denis Andrade, Ariovaldo Neto) - sistemática, biologia, comportamento e fisiologia.

- UFABC/ Campus Santo André: Centro de Ciências Naturais e Humanas (André Eterovic) - história natural, conservação e ecologia.

- UNICAMP/Campinas: Museu de Zoologia "Adão José Cardoso" (Ivan Sazima) - História natural e taxonomia.

- UNIFESP/Campus Diadema: (Ricardo J. Sawaya, Katia Pellegrino e José Eduardo de Carvalho) - taxonomia, morfologia, ecologia, biogeografia, evolução, conservação e fisiologia.

- USP/São Paulo: Departamento de Ecologia (Marcio R. C. Martins) - ecologia, morfologia, evolução e conservação.

- USP/São Paulo: Departamento de Zoologia (Miguel T. Rodrigues) - sistemática e filogenia.

- USP/São Paulo: Museu de Zoologia (Hussam Zaher) - sistemática e filogenia.

- USP/São Paulo: Departamento de Fisiologia (Carlos Navas) - fisiologia evolutiva, ecofisiologia e biologia termal.

- USP/Piracicaba: Escola Superior de Agricultura Luiz de Queiroz (ESALQ) (Luciano Verdade) - ecologia aplicada e manejo de fauna.

\section{Perspectivas}

Inventários intensivos sobre a fauna de répteis do estado, especialmente aquela ainda situada em áreas não amostradas, constituem a base para estudos mais avançados. Somente no campo poderemos detectar espécies ainda desconhecidas e restritas a tipos particulares de paisagens, já praticamente desaparecidos, mas ainda fundamentais para a compreensão da história ecológica e evolutiva dos ecossistemas do estado. Estas informações permitirão enriquecer nossa base de dados georeferenciados e abrirão espaço para estudos mais refinados de modelagem biogeográfica. O elevado poder preditivo das técnicas de modelagem, quando associado aos estudos de filogeografia molecular, permitirá avanços expressivos no detalhamento dos processos históricos, genéticos e demográficos que levaram à estruturação das populações atuais de répteis do estado, permitindo assim uma elaboração mais refinada de alvos e estratégias de conservação.

Estudos mais abrangentes sobre as relações filogenéticas e distribuição geográfica dos diferentes grupos de espécies, gêneros, e categorias hierárquicas superiores de répteis, visando descrever de modo mais acurado os seus padrões históricos de diversificação, representam área prioritária de pesquisa. Será somente através do estudo nestas várias escalas temporais que poderemos compreender melhor a dinâmica histórica dos espaços naturais do estado ao longo do tempo. Esses dados, quando disponíveis, poderão então ser empregados na implementação efetiva de medidas de conservação da diversidade dos répteis que ocorrem no Estado de São Paulo.

\section{Agradecimentos}

Agradecemos aos seguintes colegas por compartilharem informação sobre os répteis do Estado de São Paulo: Fausto P. Campos, Felipe F. Curcio, Flavio Molina, Francisco L. Franco, Giuseppe Puorto, Hebert Ferrarezzi, José Cassimiro da Silva Junior, Mauro Teixeira JUNIOR, Otávio A. V. Marques, Paulo Roberto Manzani, Pedro Bernardo, Renato Recoder, Ricardo G. Fuentes, Síria 
Ribeiro, Valdir J. Germano e Vivian Trevine. Agradecemos ainda os seguintes colegas pela ajuda na compilação das bases de dados e bibliografia: Amon Mendes, Ana Paula Suarez, Carolina C. Mello, Fernando M. Couto (in memoriam), Hana Suzuki, Sérgio Serrano, Priscylla Moll e Thiago Pires. Agradecemos a João Giovanelli pelo auxílio na confecção da análise de lacunas de amostragem. RJS agradece à FAPESP (proc. 2008/54472-2) e INCTTOX pelo apoio financeiro. Este estudo também foi financiado pela Fundação de Amparo à Pesquisa do Estado de São Paulo através de um Projeto Temático BIOTA/FAPESP concedido a HZ (número 2002/13602-4). H. Zaher, M. T. Rodrigues e R. J. Sawaya são bolsistas do CNPq. C. Nogueira é bolsista CAPES-PNPD.

\section{Referências Bibliográficas}

ÁVILA-PIRES, T.C.S. 1995. Lizards of Brazilian Amazonia (Reptilia: Squamata). Zoologische Verhandelingen Leiden 299: 1-706.

ARAUJO, C.O., CORRÊA-FILHO, D.T. \& SAWAYA, R.J. 2010. Snake assemblage of Estação Ecológica de Santa Bárbara, SP: a Cerrado remnant in Southeastern Brazil. Biota Neotrop. 10(2): http://www.biotaneotropica. org.br/v10n2/en/abstract?inventory+bn01410022010.

BARBO, F.E. 2008. Composição, história natural, diversidade e distribuição das serpentes no município de São Paulo, SP. Dissertação de Mestrado, Universidade de São Paulo, São Paulo.

BARBO, F.E. \& SAWAYA, R.J. 2008. Amphisbaenians, municipality of São Paulo, Southeastern Brazil. Checklist, 4(1):5-11.

BÉRNILS, R.S., NOGUEIRA, C. \& SILVA, V.X. 2009. Biota minas diagnóstico do conhecimento de vertebrados: Répteis. In Biota minas diagnóstico do conhecimento sobre a biodiversidade no estado de Minas Gerais (G.M. Drummond, C.S. Martins, M.S. Greco \& F. Vieira, eds.) Belo Horizonte, p.252-278.

BÉRNILS, R. S. (org.). 2010. Brazilian reptiles - List of species. http://www. sbherpetologia.org.br/. Sociedade Brasileira de Herpetologia.

BOUR, R. \& ZAHER, H. 2005. A new species of Mesoclemmys, from the open formations of Northeastern Brazil (Chelonii, Chelidae). Pap. Avul. Zool., 45(24):295-311. http://dx.doi.org/10.1590/S0031-10492005002400001

BRAZAITIS, P. 1973. The identification of living crocodiles. Zoologica (N.Y.) 58:59-101

BUSACK, S.D. \& PANDYA, S. 2001 Geographic variation in Caiman crocodilus and Caiman yacare (Crocodilia: Alligatoridae): Systematic and legal implication. Herpetologica 57(3):294-312.

CASTRO-MELLO, C. 2003. Nova espécie de Bronia Gray 1845, do estado do Tocantins, Brasil (Squamata: Amphisbaenidae). Pap. Avul. Zool., 43(7):139-143. http://dx.doi.org/10.1590/S0031-10492003000700001

CARRASCO, P.A., HARVEY, M.B. \& MUÑOZ-SARAVIA, A. 2009. The rare Andean pitviper Rhinocerophis jonathani (Serpentes: Viperidae: Crotalinae): redescription with comments on its systematics and biogeography. Zootaxa 2283: 1-15.

CARRASCO, P.A., LEYNAUD, G.C. \& SCROCCHI, G.J. 2010.Redescription of the southernmost snake species, Bothrops ammodytoides (Serpentes: Viperidae: Crotalinae). Amphibia-Reptilia 31: 323-338. http://dx.doi. org/10.1163/156853810791769491

CICCHI, P.J.P., SENA, M.A., PECCININI-SEALE, D.M. \& DUARTE, M.R. 2007. Serpentes das ilhas costeiras do estado de São Paulo, Sudeste do Brasil. Biota Neotrop. 7(2): http://www.biotaneotropica.org.br/v7n2/pt/ abstract?article+bn03907022007 (último acesso em: 25/11/2009).

COLLI, G.R., CALDWELL, J.P., COSTA, G.C., GAINSBURY, A.M., GARDA, A.A., MESQUITA, D.O., FILHO, C.M.M.R., SOARES, A.H.B., SILVA, V.N., VALDUJO, P.H., VIEIRA, G.H.C., VITT, L.J., WERNECK, F.P., WIEDERHECKER, H.C. \& ZATZ, M.G. 2003. A new species of Cnemidophorus (Squamata, Teiidae) from the Cerrado biome in central Brazil. Occasional Papers, Sam Noble Oklahoma Mus. Nat. Hist. 14:1-14.
CONDEZ, T.H.; SAWAYA, R.J. \& DIXO, M. 2009. Herpetofauna dos remanescentes de Mata Atlântica da região de Tapiraí e Piedade, SP, sudeste do Brasil. Biota Neotrop. 9:1-29. http://dx.doi.org/10.1590/ S1676-06032009000100018

CONRAD, J. 2008. Phylogeny and systematics of Squamata (Reptilia) based on morphology. Bull. Amer. Mus. Nat. Hist. 310:1-182. http://dx.doi. org/10.1206/310.1

CRESSIE, N.A.C. 1991. Statistics for spatial data. New York: John Wiley \& Sons Ed., 920p.

DORNAS, T. 2009. Compilação dos registros de quelônios, crocodilianos e aves do estado do Tocantins: biodiversidade e lacunas de conhecimento. Tese de Mestrado em Ciências do Ambiente. Universidade Federal do Tocantins. Palmas, TO, Brasil. 244 p.

EKEN, G., BENNUN, L., BROOKS, T.M., DARWALL, W., FISHPOOL, L.D.C., FOSTER, M., KNOX, D., LANGHAMMER, P., MATIKU, P., RADFORD, E., SALAMAN, P., SECHREST, W., SMITH, M.L., SPECTOR, S. \& TORDOFF, A. 2004. Key biodiversity areas as site conservation targets. Bioscience 54:1110-1118. http://dx.doi. org/10.1641/0006-3568(2004)054[1110:KBAASC]2.0.CO;2

ERNST, C. H., \& BARBOUR, R.W. 1989. Turtles of the World. Smithsonian Institution Press, Washington, D.C. and London. 313 pp.

ETEROVIC, A. \& DUARTE, M.R. 2002. Exotic snakes in Sao Paulo City, southeastern Brazil: Why xenophobia? Biodiv. Conserv. 11(2): 327-339. http://dx.doi.org/10.1023/A:1014509923673

FENWICK, A.M., GUTBERLET JUNIOR, R.L., EVANS, J.A. \& PARKINSON, C.L. 2009. Morphological and molecular evidence for phylogeny and classification of South American pitvipers, genera Bothrops, Bothriopsis, and Bothrocophias (Serpentes: Viperidae). Zool. J. Linn. Soc.156: 617-640. http://dx.doi.org/10.1111/j.1096-3642.2008.00495.x

FORLANI, M.C., BERNARDO, P.H., HADDAD, C.F.B. \& ZAHER, H. 2010. Herpetofauna do Parque Estadual Carlos Botelho, São Paulo, Brasil. Biota Neotrop. 10(3): http://www.biotaneotropica.org.br/v10n3/ pt/abstract?article+bn00210032010 (último acesso em 01/07/2010).

FROST, D.R. \& HILLIS, D.M. 1990. Species in concept and practice: herpetological applications. Herpetologica 46:87-104.

FROST, D.R., ETHERIDGE, R., JANIES, D. \& TITUS, T.A. 2001. Total evidence, sequence alignment, evolution of polychrotid lizards, and a reclassification of the Iguania (Squamata: Iguania). Amer. Mus. Nov., 3343:1-38.

GAMBLE, T., BAUER, A.M., GREENBAUM, E. \& JACKMAN, T.R. 2008. Out of the blue: a novel, trans-Atlantic clade of geckos (Gekkota, Squamata). Zool. Scripta 37(4):355-366. http://dx.doi.org/10.1111/ j.1463-6409.2008.00330.x

GASTON, K.J. 1996. Species-range-size distributions: patterns, mechanisms and implications. Trends Ecol. Evol. 11:197-201. http://dx.doi. org/10.1016/0169-5347(96)10027-6

GIBBONS, J.W., SCOTT, D.E., RYAN, T.J., BUHLMANN, K.A., TUBERVILLE, T.D., METTS, B.S., GREENE, J.L. MILLS, T., LEIDEN, Y., POPPY, S. \& WINNE, C.T. 2000. The Global Decline of Reptiles, Déjà Vu Amphibians. BioScience 50:653-666.

HARTMANN, P.A., HARTMANN, M.T. \& MARTINS, M. 2009. Ecology and natural history of a snake assemblage at Núcleo Santa Virgínia, Parque Estadual da Serra do Mar, southeastern Brazil. Biota Neotrop., 9(3) : http:// www.biotaneotropica.org.br/v9n3/en/abstract?article+bn03609032009 (último acesso em 01/07/2010).

HIJMANS, R.J., GUARINO, L., CRUZ, M. \& ROJAS, E. 2001. Computer tools for spatial analysis of plant genetic resources data: 1. DIVA-GIS. Plant Gen. Res. Newsl. 127:15-19.

HOOGMOED, M.S. \& GRUBER, U. 1983. Spix and Wagler type specimens of reptiles and amphibians in the Natural History Museum in Munich (Germany) and Leiden (The Netherlands). Spixiana 9: 319-415.

IBAMA. 2008. Lista Nacional das Espécies da Fauna Brasileira Ameaçadas de Extinção. (Acessível em: http://www.mma.gov.br/port/sbf/fauna/index.cfm). 
KRONKA, F.N., NALON, M.A., MATSUKUMA, C.K., KANASHIRO, M.M., YWANE, M.S.S., PAVÃO, M., DURIGAN, G., LIMA, L.M.P.R., GUILLAUMON, J.R., BAITELLO, J.B., BORGO, S.C., MANETTI, L.A., BARRADAS, A.M.F., FUKUDA, J.C., SHIDA, C.N., MONTEIRO, C.H.B., PONTINHA, A.A.S., ANDRADE, G.G., BARBOSA, O., SOARES, A.P., JOLY, C.A. \& COUTO, H.T.Z. 2005. Inventário florestal da vegetação natural do Estado de São Paulo. Secretaria do Meio Ambiente, Instituto Florestal - Imprensa Oficial, São Paulo. 200 pp.

MARQUES, O.A.V. 1998. Composição faunística, história natural e ecologia de serpentes da mata atlântica, na região da Estação Ecológica JuréiaItatins. Tese de Doutorado Universidade de São Paulo, São Paulo.

MARQUES, O.A.V., MARTINS, M. \& ABE, A.S. 1998. Estudo diagnóstico da diversidade de répteis do Estado de São Paulo. In Biodiversidade do Estado de São Paulo: Síntese do conhecimento ao final do século XX (R.M.C. Castro, org). FAPESP, São Paulo, p. 29-38.

MARQUES, O. A. V., SAWAYA, R. J., STENDER-OLIVEIRA, F. \& FRANÇA, F.G.R. 2006. Ecology of the colubrid snake Pseudablabes agassizii in south-eastern South America. Herp. J. 16: 37-45.

MARQUES, O.A.V., NOGUEIRA, C., SAWAYA, R.J., BÉRNILS, R.S, MARTINS, M., MOLINA, F., FERRAREZZI, H., FRANCO, F.L. \& GERMANO, V.J. 2009a. Répteis. In Livro Vermelho da Fauna Ameaçada de Extinção do Estado de São Paulo São Paulo: SEMA (C. KIERULFF. Org.), p. 285-327.

MARQUES, O.A.V., PEREIRA, D.N., BARBO F.E., GERMANO, V.J. \& SAWAYA, R.J. 2009b. Reptiles in São Paulo municipality: diversity and ecology of the past and present fauna. Biota Neotrop., 9(2): http://www. biotaneotropica.org.br/v9n2/en/abstract?article+bn02309022009 (último acesso em: 01/03/2009)

METZGER, J.P., RIBEIRO, M.C., CIOCHETI, G. \& TAMBOSI, L.R. 2008 Uso de índices de paisagem para a definição de ações. In Diretrizes para a conservação e restauração da biodiversidade no estado de São Paulo. (R.R. Rodrigues \& V.L.R. Bononi, orgs.). FAPESP, São Paulo.

MINISTÉRIO DO MEIO AMBIENTE - MMA. 2003. Lista das espécies da fauna brasileira ameaçada de extinção. Instrução normativa no. 3 de 27/mai/2003. Brasília, DF.

MIRALLES, A. \& CARRANZA, S. 2010. Systematics and biogeography of the Neotropical genus Mabuya, with special emphasis on the Amazonian skink Mabuya nigropunctata (Reptilia, Scincidae). Mol. Phylogenet. Evol. 54(3):857-869. PMid:19874906. http://dx.doi.org/10.1016/j. ympev.2009.10.016

MOTT, T. \& VIEITES, D.R. 2009. Molecular phylogenetics reveals extreme morphological homoplasy in Brazilian worm lizards challenging current taxonomy. Mol. Phyl. Evol., 51: 190-200. PMid:19405188. http://dx.doi. org/10.1016/j.ympev.2009.01.014

MYERS, C.M. 2009. Comment on the proposed precedence of Anolis chrysolepis Duméril \& Bibron, 1837 (Reptilia: Squamata) over Draconura nitens Wagler, 1830. Bul.Zool. Nom. 76(1): 788.

PELLEGRINO, K.C.M., RODRIGUES, M.T., YONENAGA-YASSUDA, Y. \& SITES, J.W. 2001. A molecular perspective on the evolution of microteiid lizards (Squamata: Gymnophthalmidae) and a new classification for the family. Biol. J. Lin. Soc. 74:315-338. http://dx.doi. org/10.1111/j.1095-8312.2001.tb01395.x

PEREZ, R. \& RIBEIRO, S.L.B. 2008. Reptilia, Squamata, Amphisbaenidae, Leposternon spp.: Distribution extension, new state record, and geographic distribution map. Check List (4):291-294.

POUGH, F.H., ANDREWS, R.M., CADLE, J.E., CRUMP, M.L., SAVITSKY, A.H. \& WELLS, K.D. 2004. Herpetology. Pearson Prentice-Hall, Upper Saddle River. $726 \mathrm{p}$
RAXWORTHY, C.J., MARTINEZ-MEYER, E., HORNING, N., NUSSBAUM, R.A., SCHNEIDER, G.E., ORTEGA-HUERTA, M.A. \& PETERSON, A.T. 2003. Predicting distributions of known and unknown reptile species in madagascar. Nature 426: 837-841. PMid:14685238.

REBOUÇAS-SPIEKER, R. 1981a. Sôbre uma espécie de Mabuya do Nordeste do Brasil (Sauria, Scincidae). Pap. Avul. Zool. 34(9): 121-123.

REBOUÇAS-SPIEKER, R. 1981b. Sobre uma nova espécie de Mabuya da Amazônia brasileira (Sauria, Scincidae). Pap. Avul. Zool. 34(16):161-163.

RIBEIRO, S., CASTRO-MELLO, C. \& NOGUEIRA, C. 2009. New species of Anops Bell, 1833 (Squamata, Amphisbaenia) from Jalapão Region in the Brazilian Cerrado. J. Herp. 43:21-28. http://dx.doi.org/10.1670/07299R1.1

RODRIGUES, M.T. 1986. Uma nova espécie de Phyllopezus de Cabaceiras: Paraiba: Brasil, com comentarios sobre a fauna de lagartos da área (Sauria, Gekkonidae). Pap. Avul. Zoo. 36(20):237-250.

RODRIGUES, M.T., PAVAN, D.T. \& CURCIO, F.F. 2007. Two new species of lizards of the genus Bachia (Squamata, Gymnophthalmidae) from Central Brazil. J. Herp. 41: 545-553. http://dx.doi.org/10.1670/06-103.1

RODRIGUES, M. T., CAMACHO, A., NUNES, P.M.S., RECODER, R.S., TEIXEIRA JUNIOR, M., VALDUJO, P.H., GHELLERE, J.M., MOTT, T. \& NOGUEIRA, C. 2008. A new species of the lizard genus Bachia from the Cerrados of Central Brazil. Zootaxa 1875:39-50.

ROSSA-FERES, D.C., MARTINS, M., MARQUES, O.A.V., MARTINS, I.A., SAWAYA, R.J. \& HADDAD, C.F.B. 2008. Herpetofauna, p. 82-98, In Diretrizes para a conservação e restauração da biodiversidade no estado de São Paulo. (R.R. Rodrigues \& V.L.R. Bononi, orgs.). São Paulo, FAPESP.

SAWAYA, R.J., MARQUES, O.A.V. \& MARTINS, M. 2008. Composição e história natural das serpentes do Cerrado de Itirapina, São Paulo, sudeste do Brasil. Biota Neotrop. 8(2):http://www.biotaneotropica.org.br/v8n2/ pt/abstract?inventory+bn01308022008. (último acesso em 01/07/2010).

UETZ, P. 2010. The Reptile Database. http://www.reptile-database.org/.

UZZELL, T.M. 1959. Teiid lizards of the genus Placosoma. Oc. Pap. Mus. Zool., University of Michigan 606: 1-16.

VANZOLINI, P.E. 1948. Notas sobre os ofídios e lagartos da Cachoeira de Emas, no municipio de Pirassununga, estado de Sao Paulo. Rev. Bras. Biol. 8: 377-400.

VANZOLINI, P.E. 1981a. The scientific and political contexts of the Bavarian Expedition to Brazil. In J.B. Von Spix \& J. G. Wagler Herpetology of Brazil. SSAR Facs. Repr. In Herpet.: ix-xxix.

VANZOLINI, P.E. 1981b. A quasi-historical approach to the natural history of the differentiation of reptiles in tropical geographic isolates. Pap. Avul Zool. 34(19):189-204.

VANZOLINI, P.E. \& WILLIAMS, E.E. 1970. South American anoles: the geographic differentiation and evolution of the Anolis chrysolepis species group (Sauria, Iguanidae). Arq. Zool. 19: 1-298.

VANZOLINI, P.E. \& WILLIAMS, E.E. 1981. The vanishing refuge: A mechanism for ecogeographic speciation. Pap. Avul. Zool. 34: 251-260.

WHITING, A.S., SITES JUNIOR, J.W., PELLEGRINO, K.C.M. \& RODRIGUES, M.T. 2006. Comparing alignment methods for inferring the history of the new world lizard genus Mabuya (Squamata: Scincidae). Mol. Phylogenet. Evol. 38(3):719-730.

WILLIAMS, E.E. \& VANZOLINI, P.E. 1980. Notes and biogeographic comments on anoles from Brasil. Pap. Avul. Zool. 34(6): 99-108.

ZAHER, H., GRAZZIOTIN, F.G., CADLE, J.A., MURPHY, R.W., MOURA-LEITE, J.C. \& BONATTO, S.L. 2009. Molecular phylogeny of advanced snakes (Serpentes,Caenophidia) with an emphasis on South AmericanXenodontines: a revised classification and descriptions of new taxa. Pap. Avul. Zool. 49(11): 115-153. http://dx.doi.org/10.1590/S003110492009001100001 


\section{Apêndice}

Apêndice 1. Lista de projetos no âmbito ou indiretamente relacionados ao Programa BIOTA/FAPESP, que contribuíram para o diagnóstico da riqueza e distribuição de espécies de répteis no Estado de São Paulo. Os projetos estão ordenados pelo nome do coordenador.

Appendix 1. List of projects directly or indirectly related to the BIOTA/FAPESP Program that contributed with the production of the diagnostic on the reptilian species richness and distribution in the state of São Paulo.

\begin{tabular}{|c|c|c|c|}
\hline & Projeto & Coordenador & Instituição sede \\
\hline 1 & $\begin{array}{l}\text { Evolução da fauna de répteis no sudeste brasileiro do } \\
\text { Cretáceo Superior ao recente: paleontologia, filogenia } \\
\text { e biogeografia }\end{array}$ & Hussam E. D. Zaher & $\begin{array}{l}\text { Museu de Zoologia da USP/ } \\
\text { São Paulo }\end{array}$ \\
\hline 2 & $\begin{array}{l}\text { A fauna de vertebrados terrestres da área sob influência } \\
\text { das Linhas de Transmissão Itaberá-Tijuco Preto III(SP) }\end{array}$ & Miguel T. Rodrigues & USP/São Paulo \\
\hline 3 & $\begin{array}{l}\text { A fauna de vertebrados terrestres da área sob influência } \\
\text { das Linhas de Transmissão Ivaiporã-Itaberá (SP/PR) }\end{array}$ & Miguel T. Rodrigues & USP/São Paulo \\
\hline 4 & $\begin{array}{l}\text { Sistemática, ecologia e Evolução de Répteis e Anfíbios } \\
\text { Neotropicais }\end{array}$ & Miguel T. Rodrigues & USP/São Paulo \\
\hline 5 & $\begin{array}{l}\text { Historia natural de duas especies simpatricas de } \\
\text { Enyalius (Squamata: Leiosauridae) na Estação } \\
\text { Biológica de Boraceia, São Paulo }\end{array}$ & Miguel T. Rodrigues & USP/São Paulo \\
\hline 6 & $\begin{array}{l}\text { Diversidade e Conservação da Fauna de Répteis do } \\
\text { sudeste da Floresta Atlântica }\end{array}$ & Denise Peccinini-Seale & USP/São Paulo \\
\hline 7 & $\begin{array}{l}\text { Conservação de anfíbios e répteis neotropicais: } \\
\text { aspectos ecológicos e evolutivos }\end{array}$ & Márcio R. C. Martins & USP/São Paulo \\
\hline 8 & $\begin{array}{l}\text { História Natural Ecologia e Evolução de Vertebrados } \\
\text { Brasileiros }\end{array}$ & Márcio R. C. Martins & USP/São Paulo \\
\hline 9 & $\begin{array}{l}\text { Diversidade, distribuição e conservação da } \\
\text { herpetofauna do Estado de São Paulo }\end{array}$ & Ricardo J. Sawaya & Instituto Butantan/São Paulo \\
\hline
\end{tabular}

Apêndice 2. Referências Bibliográficas Adicionais.

Appendix 2. Additional bibliographic references.

AGUIAR-De-DOMENICO, E. 2008. Herpetofauna do Mosaico de Unidades de Conservação do Jacupiranga (SP). Dissertação de Mestrado, Universidade de São Paulo, São Paulo.

BARBO, F.E. 2008. Os Répteis no Município de São Paulo: aspectos históricos, diversidade e conservação. In Além do Concreto: contribuições para a proteção da biodiversidade paulistana (L.R. Malagoli, F.B. Bajestero \& M. Whately, eds.). Editora Instituto Socioambiental, São Paulo, p.234-267.

BÉRNILS, R. S. 2009. Composição e padrões de distribuição de Caenophidia (Squamata, Serpentes) das Serras Atlânticas e Planaltos do Sudeste da América do Sul. Tese de Doutorado. 832 p. Museu Nacional. Rio de Janeiro, Universidade Federal do Rio de Janeiro.

CADLE, J.E. \& GREENE, H.W. 1993. Phylogenetic patterns, biogeography, and the ecological structure of neo-tropical snake assemblages. In Species diversity in ecological communities: historical and geographic perspectives (R.E. Ricklefs \& D. Schluter, eds.). University of Chicago Press, Illinois, p.281-293.

CAMOLEZ, T. \& ZAHER, H. 2010. Levantamento, identificação e descrição da fauna de Squamata do Quaternário brasileiro (Lepidosauria). Arq. Zool. $46:$ 1-96.

CENTENO, F. 2008. Diversidade e uso do ambiente pelos anfíbios e répteis da Ilha de São Sebastião, Ilhabela, SP. Dissertação de Mestrado, Universidade de São Paulo, São Paulo.

CENTENO, F.C., SAWAYA, R.J. \& MARQUES, O.A.V. 2008. Snake assemblage of Ilha de São Sebastião, southeastern Brazil: comparison to mainland. Biota Neotrop. 8(3): http://www.biotaneotropica.org.br/v8n3/en/abstract?article+bn00608032008 (último acesso em 25/11/2009).

CENTENO, F.C., SAWAYA, R.J. \& GERMANO, V.J. 2010. A new species of Liotyphlops (Serpentes: Anomalepididae) from the Atlantic Coastal Forest in southeastern Brazil. Herpetologica 66: 86-91. http://dx.doi.org/10.1655/08-079.1

CICCHI, P.J.P., SERAFIM, H., SENA, M.A., CENTENO, F.C. \& JIM, J. 2009. Herpetofauna em uma área de Floresta Atlântica na Ilha Anchieta, município de Ubatuba, sudeste do Brasil. Biota Neotrop. 9(2): http://www.biotaneotropica.org.br/v9n2/pt/abstract?inventory+bn01009022009 (último acesso em: 25/11/2009).

DALMOLIN, P.C. 2002. Composição e história natural da comunidade de serpentes da estação ecológica de Jataí e outras áreas do município de Luis Antonio, SP. Dissertação de mestrado, Universidade Federal de São Carlos, São Carlos.

DIXO, M. 2005. Diversidade de sapos e lagartos de serrapilheira numa paisagem fragmentada do Planalto Atlântico de São Paulo. Tese de Doutorado, Universidade de São Paulo, São Paulo.

DIXO, M. \& VERDADE, V.K. 2006. Herpetofauna de serapilheira da Reserva Florestal do Morro Grande, Cotia (SP). Biota Neotrop. 6(2): http://www. biotaneotropica.org.br/v6n2/pt/abstract?article+bn00706022006 (último acesso em 10/09/2009).

FORLANI, M.C., BERNARDO, P.H., HADDAD, C.B.F. \& ZAHER, H. 2010. Herpetofauna do Parque Estadual Carlos Botelho, São Paulo, Brasil. Biota Neotrop. 10(3): http://www.biotaneotropica.org.br/v10n3/pt/abstract?article+bn00210032010 (último acesso em 01/07/2010).

HARTMANN, P.A. 2005. História natural e ecologia de duas taxocenoses de serpentes na Mata Atlântica. Tese de Doutorado, Universidade Estadual Paulista "Júlio de Mesquita Filho", Rio Claro.

HARTMANN, P.A., HARTMANN, M.T. \& MARTINS, M. 2009. Ecology of a snake assemblage in the Atlantic Forest of southeastern Brazil. Pap. Avul. Zool., 49(27): 343-360. http://dx.doi.org/10.1590/S0031-10492009002700001 
MARQUES, O.A.V. \& SAZIMA, I. 2004. História natural dos répteis da Estação Ecológica Juréia-Itatins. In Estação Ecológica Juréia-Itatins: Ambiente Físico, Flora e Fauna (O.A.V. Marques \& W. Duleba, eds.). Ed. Holos, Ribeirão Preto, p. 257-277.

MARQUES, O.A.V., ETEROVIC, A. \& SAZIMA, I. 2004. Snakes of the Brazilian Atlantic forest: an illustrated field guide for the Serra do Mar Range. Editora Holos, Ribeirão Preto.

MIRALLES, A \& CARRANZA, S. 2010. Systematics and biogeography of the Neotropical genus Mabuya, with special emphasis on the Amazonian skink Mabuya nigropunctata (Reptilia, Scincidae). Mol. Phyl. Evol. 54 (3): 857-869. http://dx.doi.org/10.1016/j.ympev.2009.10.016

NOGUEIRA, C. 2006. Diversidade e padrões de distribuição da fauna de lagartos do Cerrado. Tese de Doutorado, Universidade de São Paulo, São Paulo.

NOGUEIRA, C., COLLI, G.R., COSTA, G.C. \& MACHADO, R.B. 2010. Diversidade de répteis Squamata e evolução do conhecimento faunístico no Cerrado. Pages 329-372. In Cerrado: conhecimento científico quantitativo como subsídio para ações de conservação (I. R. Diniz, J. Marinho-Filho, R. B. Machado \& R. B. Cavalcanti, eds). Brasília: Thesaurus Editora, 2010.

PEREZ, R. \& RIBEIRO, S.L.B. 2008. Reptilia, Squamata, Amphisbaenidae, Leposternon spp.: Distribution extension, new state record, and geographic distribution map. Checklist 4 (3): 291-294.

ROCHA, C.F.D., BERGALLO, H.G., CONDE, C.F.V., BITTENCOURT, E.B. \& SANTOS, H.C. 2008. Richness, abundance, and mass in snake assemblages from two Atlantic Rainforest sites (Ilha do Cardoso, São Paulo) with differences in environmental productivity. Biota Neotrop. 8(3): http://www.biotaneotropica. org.br/v8n3/en/abstract?article+bn01408032008 (último acesso em 01/07/2010).

SAWAYA, R.J. 2004. História natural e ecologia das serpentes de Cerrado da região de Itirapina, SP. Tese de Doutorado, Universidade Estadual de Campinas, Campinas.

SAZIMA, I. \& HADDAD, C.F.B. 1992. Répteis da Serra do Japi: notas sobre história natural. In História natural da Serra do Japi: ecologia e preservação de uma área florestal no sudeste do Brasil. (P. Morellato, org.). Editora UNICAMP, Campinas, p. 212-235.

SAZIMA, I. \& MANZANI, P.R. 1995. As cobras que vivem numa reserva florestal urbana. In Ecologia e preservação de uma floresta tropical urbana: Reserva de Santa Genebra. (P.C. Morellato \& H.F. Leitão-Filho, eds). Editora UNICAMP, Campinas, p. 78-82.

SAZIMA, I. 2001. Répteis. In Intervales: Fundação para a conservação e produção florestal do estado de São Paulo (C. Leonel, ed.). Editora Secretaria do Meio Ambiente/Fundação Florestal, São Paulo, p.148-158.

SENA, M.A. 2007. Levantamento da fauna e estudo cromossômico de algumas espécies de Reptilia, Squamata, do município de Cananéia, SP. Dissertação de Mestrado, Universidade de São Paulo, São Paulo.

THOMÉ, M.T.C. 2006. Diversidade de anuros e lagartos em fisionomias de Cerrado na região de Itirapina, Sudeste do Brasil. Dissertação de Mestrado, Universidade de São Paulo, São Paulo.

VASCONCELOS, T.S. \& SANTOS, T.G. Reptilia, Serpentes, Dipsadidae, Phalotris lativittatus: Distribution extension and geographic distribution map. Checklist 5(1): 5-7.

WHITING, A.S., SITES JUNIOR, J.W., PELLEGRINO, K.C.M. \& RODRIGUES, M.T. 2006. Comparing alignment methods for inferring the history of the new world lizard genus Mabuya (Squamata: Scincidae). Mol. Phyl. Evol. 38:719-730. PMid:16364664. http://dx.doi.org/10.1016/j.ympev.2005.11.011

ZAHER, H. 1996. A new genus and species of Pseudoboine snake, with a revision of the genus Clelia (Serpentes, Xenodontinae). Boll. Mus. Reg. Sci. Nat. Torino 14(2): 289-337. 